\title{
Optimal Centering, Tolerancing, and Yield Determination via Updated Approximations and Cuts
}

\author{
JOHN W. BANDLER, FELLOW, IEEE, AND HANY L. ABDEL-MALEK, STUDENT MEMBER, IEEE
}

\begin{abstract}
This paper presents a new approach to optimal design centering, the optimal assignment of parameter tolerances and the determination and optimization of production yield. Based upon multidimensional linear cuts of the tolerance orthotope and uniform distributions of outcomes between tolerance extremes in the orthotope, exact formulas for yield and yield sensitivities, with respect to design parameters, are derived. The formulas employ the intersections of the cuts with the orthotope edges, the cuts themselves being functions of the original design constraints. Our computational approach involves the approximation of all the constraints by low-order multidimensional polynomials. These approximations are continually updated during optimization. Inherent advantages of the approximations which we have exploited are that explicit sensitivities of the design performance are not required, available simulation programs can be used, inexpensive function and gradient evaluations can be made, inexpensive calculations at vertices of the tolerance orthotope are facilitated during optimization and, subsequently, inexpensive Montc Carlo verification is possible. Simple circuit examples illustrate worst case design and design with yields of less then 100 percent. The examples also provide verification of the formulas and algorithms.
\end{abstract}

\section{INTRODUCTION}

$\mathrm{O}$ PTIMAL tolerance assignment is the process of associating the largest tolerances with design parameters to minimize cost. Design centering is the process of defining a set of nominal parameter values to maximize the tolerances or to maximize the yield for known but unavoidable statistical fluctuations. This paper integrates the concepts of design centering, the optimal assignment of parameter tolerances and the determination and optimization of production yield into an overall optimal design process.

Our computational approach should be viewed in the context of the following important work in this area: the nonlinear programming approach of Bandler et al. [1], [2] and by Pinel and Roberts [3], the branch and bound method of Karafin [4], the Monte Carlo approach of Elias [5], and the Director and Hachtel technique involving approximations of the feasible region [6]. It makes use of

Manuscript received October 13, 1976; revised June 15, 1977 and January 6, 1978. This work was supported by the National Research Council of Canada under Grant A7239. This paper is based on material presented at the 1977 IEEE International Symposium on Circuits and Systems, Phoenix, AZ, April 25-27, 1977.

J. W. Bandler is with the Group on Simulation, Optimization and Control and the Department of Electrical Engineering, McMaster University, Hamilton, Ont. L8S 4L7, Canada.

H. L. Abdel-Malek was with the Group on Simulation, Optimization and Control and the Department of Electrical Engineering, McMaster University, Hamilton, Ont. L8S 4L7, Canada. He is now with the Department of Engineering Physics and Mathematics, Faculty of Engineering, Cairo University, Giza, Egypt. approximations of all the constraints by low-order multidimensional polynomials. These approximations are continually updated in critical regions identified during optimization and integrated with the nonlinear program which inscribes an orthotope in the constraint region by minimizing a suitable scalar objective function. This orthotope will actually be the optimum tolerance region for a worst case design problem with independent variables.

The readily differentiable approximations permit efficient gradient methods of minimization to be employed as well as inexpensive calculations at vertices of the tolerance orthotope, which tend to locate the critical regions. The yield problem commences when the orthotope is allowed to expand beyond the boundary of the constraint region. Attention is then directed to the critical regions which contribute to the yield calculation.

Section II describes the nature of the tolerance problem and discusses the implications of the assumption of onedimensional convexity [7], [8]. Section III formally introduces the multidimensional polynomial. Our approach to choosing suitable interpolation base points is given. The section includes an efficient algorithm for evaluating the approximations and their derivatives at different vertices in different well-chosen interpolation regions. Section IV presents algorithms for worst case design: Phase 1 deals with a single interpolation region, and Phase 2 involves two or more interpolation regions. These interpolation regions are updated according to desired accuracy for the approximate constraints in critical regions.

Based upon multidimensional linear cuts of the tolerance orthotope and uniform distributions of outcomes between tolerance extremes in the orthotope, Section V presents exact formulas for yield and yield sensitivities with respect to design parameters. The formulas employ the intersections of the cuts with the orthotope edges, the cuts themselves being functions of the original design constraints. Ways of treating linear and quadratic constraints (actual or approximate) are discussed so that results obtained by implementing the material of the previous sections can be followed up.

Section VI details an algorithm embodying all the ideas and results of Sections II to V. It deals with optimization involving yield less than 100 percent. Appropriate approximations to the boundary based on a single function of least $p$ th type [9] within each critical region are utilized. 
Some illustrative examples are also included. A two-section quarter-wave transmission-line transformer is used to explain how a worst case design is obtained and, further, is used for yield determination and optimization. A worst case design and a well-centered design for yield less than 100 percent for a three-section low-pass $L C$ filter are included. Practical examples of nonideal two-section and three-section waveguide transformers are described.

\section{Nonlinear Programming Formulation of THE TOlerance Problem}

\section{Introductory Concepts}

An engineering design can be described by a vector of nominal parameters $\phi^{0}$ and an associated vector of manufacturing tolerances $\epsilon$, where

$$
\boldsymbol{\phi}^{0} \triangleq\left[\begin{array}{l}
\phi_{1}^{0} \\
\phi_{2}^{0} \\
\cdot \\
\cdot \\
\phi_{k}^{0}
\end{array}\right] \geqslant \mathbf{0}, \quad \epsilon \triangleq\left[\begin{array}{l}
\epsilon_{1} \\
\epsilon_{2} \\
\cdot \\
\cdot \\
\epsilon_{k}
\end{array}\right] \geqslant \mathbf{0}
$$

and where $k$ is the number of designable parameters. Accordingly, any design outcome is represented by a point which lies inside a tolerance region $R_{\epsilon}$ as shown in Fig. 1. For simplicity as well as the implications of a uniform distribution of outcomes between tolerance extremes $\phi_{i}^{0} \pm \epsilon_{i}$, we define

$$
R_{\epsilon} \triangleq\left\{\phi \mid \phi=\phi^{0}+E \mu, \mu \in R_{\mu}\right\}
$$

where

$$
R_{\mu} \triangleq\left\{\boldsymbol{\mu} \mid-1 \leqslant \mu_{i} \leqslant 1, i=1,2, \cdots, k\right\}
$$

and where $\boldsymbol{E}$ is a $k \times k$ matrix with diagonal elements set to $\epsilon_{i}$ and $\mu$ is a random vector distributed according to the joint probability distribution function of the outcomes. Any value for $\mu$ identifies a point in $R_{\epsilon}$. The tolerance region $R_{\varepsilon}$ as defined in (2) is an orthotope in the $k$-dimensional space (see Coxeter [10]). Consequently, the tolerance region will often be referred to as the tolerance orthotope. The vertices of this orthotope are the points for which all parameters are at extreme values (positive or negative extremes), i.e., $\mu_{i} \in\{-1,1\}, i=1,2, \cdots, k$. See Fig. 1. The number of these vertices is $2^{k}$ and they are, for convenience, uniquely indexed by $\phi^{r}, r \in I_{v}$, where

$$
I_{v} \triangleq\left\{1,2, \cdots, 2^{k}\right\}
$$

Thus the set of vertices is given by

$$
R_{v}=\left\{\phi^{r} \mid r \in I_{v}\right\}
$$

This numbering scheme will allow us to identify a vertex by the number $r$ only.

Let $R_{c}$ be the constraint region, illustrated in Fig. 1, defined by $m_{c}$ functions $g_{i}(\phi)$ and given by

$$
R_{c} \triangleq\left\{\phi \mid g_{i}(\phi) \geqslant 0, \text { for all } i \in I_{c}\right\}
$$

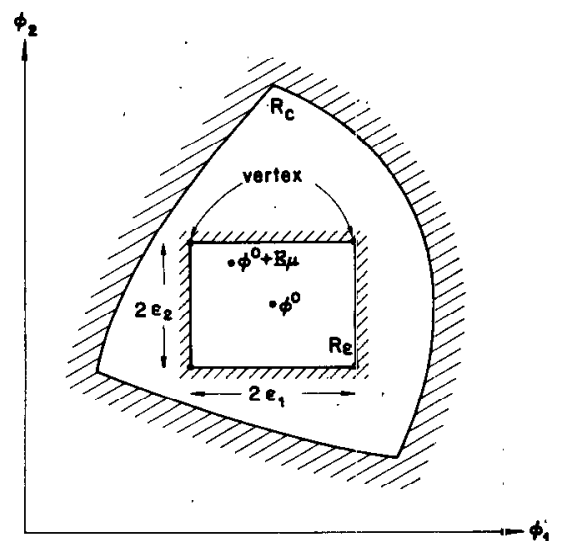

Fig. 1. Illustration of the constraint region $R_{c}$ and the tolerance region $R_{\varepsilon}$. Also, the nominal point $\phi^{0}$, an outcome $\phi^{0}+E_{\mu}$, and vertices are indicated.

where

$$
I_{c} \triangleq\left\{1,2, \cdots, m_{c}\right\} .
$$

\section{Worst Case Design}

For a worst case design [1], [2], sometimes called a design with 100 -percent yield, it is required that all design outcomes satisfy the specifications, i.e.,

$$
R_{\epsilon} \subset R_{c} \text {. }
$$

If the constraint region $R_{c}$ is one-dimensionally convex [7], it is sufficient that all vertices of $R_{\epsilon}$ belong to $R_{c}$ to guarantee that (8) is satisfied, i.e., it is sufficient to have

$$
R_{v} \subset R_{c}
$$

where, formally

$$
R_{v} \triangleq\left\{\phi \mid \phi=\phi^{0}+E \mu, \mu_{i} \in\{-1,1\}, i=1,2 ; \cdots, k\right\} .
$$

Bandler and Liu [8] and Brayton et al. [11] have considered the implications of one-dimensional convexity for certain classes of circuits.

The foregoing discussion leads to the following nonlinear programming problem for worst case design involving, in general, both centering of $\phi^{0}$ and optimal assignment of $\epsilon$ :

$$
\text { WCD } \begin{cases} & \underset{\phi^{0}, \epsilon}{\operatorname{minimize}} C\left(\phi^{0}, \epsilon\right) \\ \text { subject to } & \\ & g_{i}\left(\phi^{r}\right) \geqslant 0, \text { for all } i \in I_{c}, \text { and all } r \in I_{v}\end{cases}
$$

where $C$ is a suitable cost function and the constraints (11) are an explicit formulation of the constraint (9). The total number of constraints involved in WCD is $m_{c} \times 2^{k}$. The one-dimensional convexity assumption allowed us to have this finite number of constraints rather than the infinite number of constraints implied by ( 8 ).

Methods for solving nonlinear programs are well developed in the literature. We simply note here that efficient evaluation of the constraints, rapid determination of active constraints as well as the use of gradient techniques in 


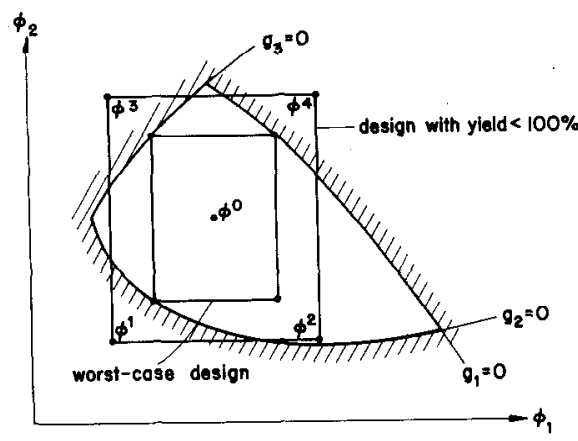

Fig. 2. Example of a worst case design and a design with yicld $<100$ percent. For the worst case design the set of active vertices is $S_{\mathrm{av}}=$ $\{1,3,4\}$. These vertices indicate critical regions where constraint violations are most likely to occur for a design with yicld $<100$ percent.

the search for the optimum values of $\phi^{0}$ and $\epsilon$ are computationally highly desirable.

The active vertices at the worst case optimum, i.e., at the solution of WCD, are those which lie on the boundary of $R_{c}$. The set of active vertices is given by

$$
S_{\mathrm{av}} \triangleq\left\{r \mid g_{i}\left(\phi^{r}\right)=0, r \in I_{v}, i \in I_{c}\right\} .
$$

See Fig. 2 for an illustration of a worst case design.

\section{Yield Less Than 100 Percent}

When the yield is allowed to drop below 100 percent we have $R_{\mathrm{\epsilon}} \not \subset R_{c}$. An appropriate nonlinear program in this case is

$$
\text { YNP }\left\{\begin{array}{c}
\underset{\phi^{0}, \epsilon}{\operatorname{minimize}} C\left(\phi^{0}, \epsilon, \mu\right) \\
\text { subject, for example, to } \\
Y\left(\phi^{0}, \epsilon, \mu\right) \geqslant Y_{L}
\end{array}\right.
$$

where $Y_{L}$ is a yield specification. A design with yield $<100$ percent is depicted in Fig. 2.

Again, this nonlinear program is to be solved for optimum values of $\phi^{0}$ and $\epsilon$. It is not necessary that all components of $\phi^{0}$ and $\epsilon$ be allowed to vary. Some of them might be fixed. The constraint on yield might be removed if the yield is represented in the cost. This case might arise, for example, if the distribution of outcomes is fixed and $\phi^{0}$ is allowed to vary in order to meet maximum yield. Although design constraints do not seem to appear explicitly in YNP they are all implicitly accounted for in the consideration of yield.

\section{Approximations to $\boldsymbol{R}_{\boldsymbol{c}}$}

Unlike optimization problems in which a single point is of interest, tolerances and uncertainties create a region of interest. The solution is usually characterized by several critical points or regions so that more information about the constraint region is required. Under the foregoing assumptions it seems reasonable to assume that for a high but less than 100-percent yield the active vertices determined by a worst case design will indicate regions where constraint violations are most likely (see Fig. 2). Accordingly, our interest must be directed to the active vertices as locations for centering reliable approximations to the boundary, which is the subject of the following section.

\section{Interpolation by Quadratic Polynomials}

Worst case design, yield analysis, and optimization involve a mass of calculations. Inadequate information on cost functions, component distributions, model uncertainties, etc., already hinders precise design solutions. Consequently, multidimensional approximations to design constraints appear to be a computational necessity without, it is felt, any significant sacrifice in design accuracy.

An approximate representation of a function $g(\phi)$, typically a constraint, using its values at a finite set of points $\phi$ is possible. These points are called nodes or base points. Interpolation is adopted since it is not only a simple approach to approximation but also because it requires relatively few actual function evaluations. In general, interpolation can be done by means of a linear combination of the set of all possible monomials [12], [13]. A monomial in $\phi$ of the order $m$ is given by

$$
\Phi(\phi)=\left(\phi_{1}\right)^{\gamma_{1}}\left(\phi_{2}\right)^{\gamma_{2}} \cdots\left(\phi_{k}\right)^{\gamma_{k}}, \quad \sum_{i=1}^{m} \gamma_{i}=m
$$

where the integers $\gamma_{i} \geqslant 0, i=1,2, \cdots, k$.

Since the accuracy of the approximation depends upon the size of the interpolation region, the critical parts of $R_{\epsilon}$ may not be covered by a single interpolation region. Thus the use of more than one interpolation region will be discussed.

\section{The Quadratic Polynomial}

A quadratic polynomial in $k$ variables can be written as

$$
\begin{aligned}
P(\phi)= & a_{1}\left(\phi_{1}\right)^{2}+a_{2}\left(\phi_{2}\right)^{2}+\cdots+a_{k}\left(\phi_{k}\right)^{2} \\
& +a_{k+1} \phi_{1} \phi_{2}+a_{k+2} \phi_{1} \phi_{3}+\cdots+a_{N-k-1} \phi_{k-1} \phi_{k} \\
& +a_{N-k} \phi_{1}+a_{N-k+1} \phi_{2}+\cdots+a_{N-1} \phi_{k}+a_{N}
\end{aligned}
$$

where

$$
N=(k+1)(k+2) / 2
$$

is the number of monomials and at the same time the number of the unknown coefficients $a_{1}, a_{2}, \cdots, a_{N}$. In order to find these coefficients, the values of $P(\phi)$ at $N$ base points $\phi_{b}$ are required. By setting

$$
P\left(\phi_{b}^{i}\right)=g\left(\phi_{b}^{i}\right), \quad i=1,2, \cdots, N
$$

a set of $N$ simultaneous linear equations is constructed. A solution for this system exists if the base points are degree-2 independent [14]. A set of $N$ points is said to be degree- $m$ independent if there exist no constants $\beta_{j}$, except $\beta_{j}=0, j=1,2, \cdots, N$, such that

$$
\sum_{j=1}^{N} \beta_{j} \Phi\left(\phi^{j}\right)=0
$$

where $\Phi$ is the monomial given in (14). 


\section{Selection of Base Points}

Suppose that the function $g(\phi)$ is to be approximated at a particular region in the parameter space. We identify this interpolation region $\vec{R}$ through a "center" of interpolation $\bar{\phi}$ and a step size $\delta$. We define, accordingly

$$
\bar{R} \triangleq\left\{\phi|| \phi_{i}-\bar{\phi}_{i} \mid \leqslant \delta_{i}, i=1,2, \cdots, k\right\}
$$

and require that the base points should satisfy

$$
\phi_{b}^{i} \in \bar{R}, \quad \ddot{i}=1,2, \cdots, N .
$$

This requirement is satisfied if the set of base points is given by

$$
\begin{array}{r}
{\left[\begin{array}{llll}
\boldsymbol{\phi}_{b}^{1} & \boldsymbol{\phi}_{b}^{2} & \cdots & \boldsymbol{\phi}_{b}^{N}
\end{array}\right]=\boldsymbol{D}\left[\begin{array}{llll}
\mathbf{0} & \mathbf{1}_{k} & -\mathbf{1}_{k} & \boldsymbol{B}
\end{array}\right]} \\
+\left[\begin{array}{llll}
\overline{\boldsymbol{\phi}} & \overline{\boldsymbol{\phi}} & \cdots & \overline{\boldsymbol{\phi}}
\end{array}\right]
\end{array}
$$

where $D$ is a $k \times k$ diagonal matrix with elements $\delta_{i}, 0$ is the zero vector of dimension $k, 1_{k}$ is a $k \times k$ unit matrix, $B$ is a $k \times(k(k-1) / 2)$ matrix defined by

$$
\boldsymbol{B}=\left[\begin{array}{llll}
\boldsymbol{\mu}_{b}^{1} & \boldsymbol{\mu}_{b}^{2} & \cdots & \boldsymbol{\mu}_{b}^{L}
\end{array}\right]
$$

in which

$$
L=k(k-1) / 2
$$

where $\mu_{b}^{j}$ are randomly selected such that

$$
\mu_{b}^{j} \in R_{\mu}, \quad j=1,2, \cdots, L .
$$

See, for example, Fig. 3.

This choice of base points preserves one-dimensional convexity/concavity of the approximated function, since there are three base points along each axis (see Appendix).

\section{Polynomial Evaluation at Vertices}

In solving the nonlinear program WCD, the values of the constraints and their derivatives at the vertices are required. Here, we develop an efficient technique for evaluating approximations to the constraints along with their derivatives for subsequent use in conjunction with gradient optimization methods.

The technique exploits simple properties of a quadratic approximation. The following two equations are used to obtain the polynomial value and its gradients at any vertex $\boldsymbol{\phi}^{r}$ using values at another vertex $\boldsymbol{\phi}^{s}$.

$P\left(\phi^{r}\right)=P\left(\phi^{s}\right)+\left(\phi^{r}-\phi^{s}\right)^{T} \nabla P\left(\phi^{s}\right)+\frac{1}{2}\left(\phi^{r}-\phi^{s}\right)^{T} H\left(\phi^{r}-\phi^{s}\right)$

$$
\nabla P\left(\phi^{r}\right)=\nabla P\left(\phi^{s}\right)+H\left(\phi^{r}-\phi^{s}\right)
$$

where

$$
\nabla \triangleq\left[\begin{array}{c}
\partial / \partial \phi_{1} \\
\partial / \partial \phi_{2} \\
\dot{\cdot} \\
\partial / \partial \phi_{k}
\end{array}\right]
$$

and

$$
\boldsymbol{H} \triangleq \nabla \nabla^{T} \boldsymbol{P}
$$

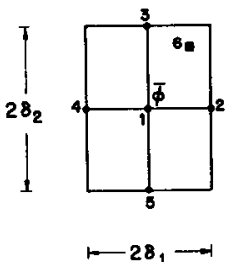

(a)

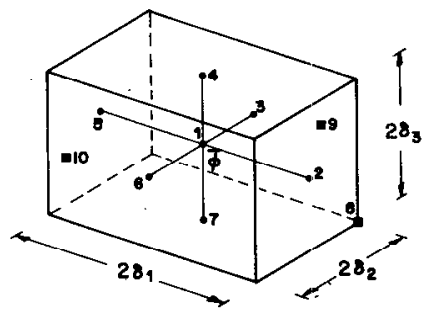

(b)

Fig. 3. Arrangement of the base points with respect to the centers of interpolation regions in (a) two dimensions (base point $\phi_{b}^{6}$ is selected randomly) and (b) three dimensions (base points $\phi_{b}^{8}, \phi_{b}^{9}$, and $\phi_{b}^{10}$ are selected randomly).

is the Hessian matrix for the quadratic approximation.

Suppose $\phi^{r}$ and $\phi^{s}$ are adjacent vertices, i.e.,

$$
\phi^{r}=\phi^{s}+2 \epsilon_{i} e_{i}
$$

where $e_{i}$ is the unit vector in the $i$ th direction. In this case (25) and (26) reduce to

$$
\begin{aligned}
P\left(\phi^{r}\right) & =P\left(\phi^{s}\right)+2 \epsilon_{i} \nabla_{i} P\left(\phi^{s}\right)+2 \epsilon_{i}^{2} H_{i i} \\
\nabla P\left(\phi^{r}\right) & =\nabla P\left(\phi^{s}\right)+2 \epsilon_{i} H_{i}
\end{aligned}
$$

where $\nabla_{i}$ is the $i$ th row of $\nabla, H_{i i}$ is the $i$ th diagonal element of $\boldsymbol{H}$, and $\boldsymbol{H}_{i}$ is the $i$ th column of $\boldsymbol{H}$.

Different approximations may be considered in different interpolation regions. To this end some relevant notation is introduced, as follows.

Let

$$
\begin{aligned}
& I_{\delta} \triangleq\left\{i \mid \delta_{i} \geqslant \epsilon_{i}\right\} \\
& I_{\epsilon} \triangleq\left\{i \mid \epsilon_{i}>\delta_{i}\right\}
\end{aligned}
$$

and the number of elements of $I_{\delta}$ and $I_{\epsilon}$ be $k_{\delta}$ and $k_{\epsilon}$, respectively. In an effort to describe the minimal number of interpolation regions $N_{\text {in }}$ which collectively contain all the vertices we consider each element of $I_{\epsilon}$ in such a way that

$$
N_{\text {in }}=2^{k_{\text {e }}}
$$

and (see, e.g., Fig. 4) that the centers of interpolation $\bar{\phi}^{l}$ are associated with $\phi^{\prime} \in R_{v}$ through

$$
\bar{\phi}^{l}=\phi^{0}+\boldsymbol{P}\left(\phi^{r}-\phi^{0}\right)
$$

where the projection matrix $\boldsymbol{P}$ is the diagonal matrix

$$
\boldsymbol{P} \triangleq\left[\begin{array}{ccccccc}
p_{1} & & & & & \\
& p_{2} & & & & \\
& & \cdot & \cdot & & \\
& & & & \cdot & \\
& & & & & p_{k}
\end{array}\right]
$$




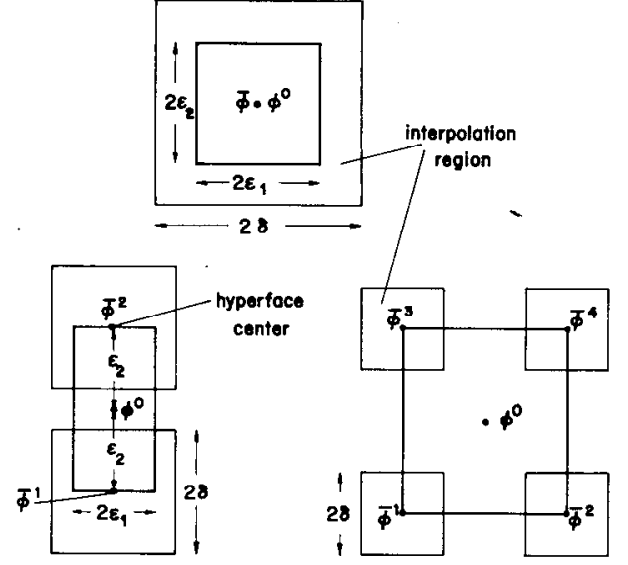

Fig. 4. Three situations created by certain step sizes $\delta=\delta_{1}=\delta_{2}$ and tolerances. The available interpolation regions and their centers are indicated as follows: (a) $I_{c}-\varnothing, I_{v}^{1}=\{1,2,3,4\}$. (b) $I_{\epsilon}=\{2\}, I_{v}^{1}=\{1,2\}$, $I_{v}^{2}=\{3,4\}$. (c) $I_{c}=\{1,2\}, I_{v}^{1}=\{1\}, I_{v}^{2}=\{2\}, I_{v}^{3}=\{3\}, I_{v}^{4}=\{4\}$.

and where

$$
p_{i}= \begin{cases}0, & i \in I_{\delta} \\ 1, & i \in I_{\epsilon}\end{cases}
$$

A suitable numbering scheme for identifying vertices is [7]

$$
r=1+\sum_{i=1}^{k}\left(\frac{\mu_{i}^{r}+1}{2}\right) 2^{i-1}, \quad \mu_{i}^{r} \in\{-1,1\}
$$

so that adjacent vertices (i.e., vertices different in the $i$ th parameter) satisfy

$$
|r-s|=2^{i-1} \text {. }
$$

An analogous numbering scheme for interpolation regions is given by

$$
l=1+\sum_{i=1}^{k} p_{i}\left(\frac{\mu_{i}^{r}+1}{2}\right) 2^{i_{i}-1}, \quad \mu_{i}^{r} \in\{-1,1\}
$$

where

$$
i_{\epsilon}=\sum_{j=1}^{i} p_{j}
$$

Intuitively, $i_{\epsilon}$ is a renumbered index derived from $i$ and the projection components $p_{j}$ to include only the elements of $I_{\epsilon}$ in such a way that a doubling of the number of interpolation regions occurs for every such element. For example, if $p_{i}=1, i=1,2, \cdots, k_{\epsilon}$ we have

$$
l=1+\sum_{i=1}^{k_{\epsilon}}\left(\frac{\mu_{i}^{r}+1}{2}\right) 2^{i-1}, \quad \mu_{i}^{r} \in\{-1,1\}
$$

since $i_{\epsilon}=i$ follows from (41).

Since a given $r$ th vertex belongs to a particular interpolation region $l$ given by (40) we can, without ambiguity, let

$$
P^{r} \triangleq P^{l}\left(\phi^{r}\right)
$$

where $P^{\prime}(\phi)$ is the polynomial constructed for the $l$ th

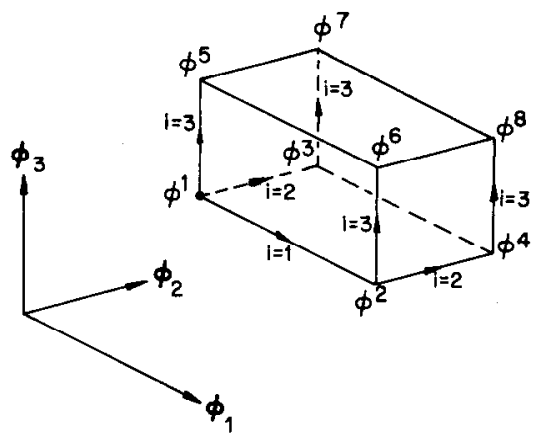

(a)

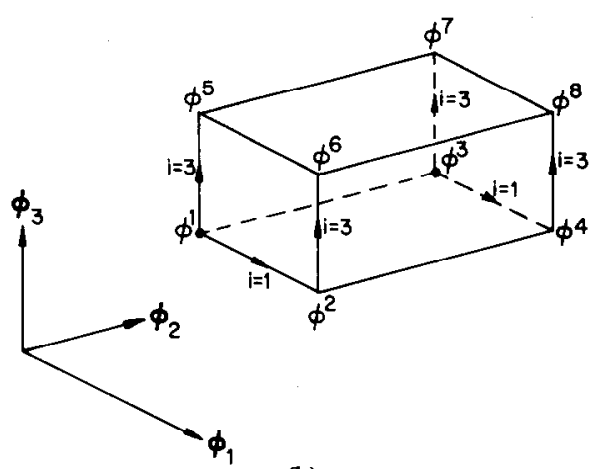

(b)

Fig. 5. Illustration of the efficient technique for evaluation of the approximations and their derivatives. (a) $k_{\delta}=3, N_{\text {in }}=1, I_{v}^{1}=I_{v}$ and, initially, $I=\{1\}$. (b) $k_{\delta}=2, N_{\text {in }}=2, I_{v}^{1}=\{1,2,5,6\}, I_{v}^{2}=\{3,4,7,8\}$ and, initially, $I=\{1,3\}$.

interpolation region. With this notation we rewrite (30) and (31) as

$$
\begin{aligned}
P^{r} & =P^{s}+2 \epsilon_{i} \nabla_{i} P^{s}+2 \epsilon_{i}^{2} H_{i i}^{l} \\
\nabla P^{r} & =\nabla P^{s}+2 \epsilon_{i} \boldsymbol{H}_{i}^{l}
\end{aligned}
$$

where the superscript $l$ identifies the relevant components of $\boldsymbol{H}$ (defined earlier) and where $r$ and $s$ are related by

$$
r=s+2^{i-1}, \quad i \in I_{\delta}
$$

implying that $\boldsymbol{\phi}^{r}$ and $\boldsymbol{\phi}^{s}$ are adjacent (see (29) and belong to the same interpolation region, viz.,

$$
r, s \in I_{v}^{l} \triangleq\left\{i \mid i \in I_{v}, \phi^{i} \in \bar{R}^{\prime}\right\}
$$

where $I_{v}$ is given by (4) and $\bar{R}^{l}$ is the $l$ th interpolation region.

\section{Algorithm for Polynomial Evaluation (APE)}

This algorithm is illustrated in Fig. 5. The figure indicates two situations in three dimensions. Polynomial and gradient evaluations are made during each iteration at corresponding vertices in certain interpolation regions, starting with one vertex per region. New vertices are systematically considered in successive iterations, their number being doubled until the candidates have been exhausted.

This algorithm assumes that quadratic polynomial values $P$ along with corresponding $\nabla P$ associated with a subset $I_{p}$ of the $N_{\text {in }}$ available interpolation regions are to be computed.. The required subset will generally be de- 
TABLE I

Computational Effort for Evaluation of the Quadratic Polynomial and Its Derivatives

\begin{tabular}{|c|c|c|}
\hline Description & Number of additions & Number of multiplications \\
\hline At one vertex only & $\frac{1}{2} k(3 k+5)$ & $\frac{3}{2} k(k+1)$ \\
\hline $\begin{array}{l}\text { At all the vertices using } \\
\text { original formula }\end{array}$ & $2^{k-1} k(3 k+5)$ & $3 \times 2^{k-1} k(k+1)$ \\
\hline $\begin{array}{l}\text { At all the vertices using } \\
\text { the efficient scheme }\end{array}$ & 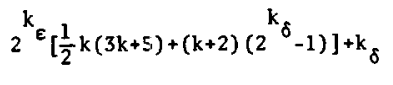 & 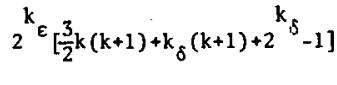 \\
\hline $\begin{array}{l}\text { At all the vertices using } \\
\text { the efficient scheme } \\
k_{\delta}=k\end{array}$ & $\frac{1}{2} k(3 k+7)+(k+2)\left(2^{k}-1\right)$ & $\frac{5}{2} k(k+1)+2^{k}-1$ \\
\hline
\end{tabular}

termined during worst case design in accordance with candidates for active vertices for the constraint under consideration.

Step 1: Evaluate $P^{s}$ and $\nabla P^{s}$ for all $s \in I$, where

$$
I=\left\{i \mid i=\min _{j \in I_{t}^{\prime}} j, l \in I_{p}\right\} .
$$

Comment: This is an initialization of the set of vertices, one vertex per interpolation region being considered, as required to start the computation of the polynomials and their gradients. Each.vertex selected is the closest possible to the origin.

Step 2: $J \leftarrow I_{\delta}$.

Comment: $J$ is a working set of indices, initialized here to correspond to all those designable parameters which can vary within each interpolation region.

Step 3: If $J=\varnothing$ stop.

Comment: This step tests whether there are any (remaining) candidates in $J$. If $J$ is empty polynomials at all the vertices within the considered interpolation regions have been evaluated.

Step 4: $i \leftarrow \min _{j \in J} j$.

Comment: This ordering process selects the index $i$ corresponding to the parameter to be varied in the following steps.

Step 5: $T \leftarrow \epsilon_{i}+\epsilon_{i}$.

Step 6: $\boldsymbol{G}_{i}^{l} \leftarrow T \boldsymbol{H}_{i}^{l}$ for all $l \in I_{p}$.

Step 7: For all $s \in I$

$$
\begin{gathered}
P^{r} \leftarrow P^{s}+T \nabla_{i} P^{s}+\epsilon_{i} G_{i i}^{l} \\
\nabla P^{r} \leftarrow \nabla P^{s}+G_{i}^{l}
\end{gathered}
$$

where $r$ and $l$ are given by (46) and (40), respectively.

Comment: The values of the polynomials and the corresponding gradient vectors are calculated at all appropriate adjacent vertices. The number of vertices at which evaluations have been made are thus doubled in this step.

Step 8: $I \leftarrow I \cup\left\{r \mid r=s+2^{i-1}, s \in I\right\}$.

Comment: The set of vertices already considered is updated.

Step 9: $J \leftarrow J \backslash\{i\}$.

Comment: The index $i$, already exploited, is removed from the working set $J$.

Step 10: Go to Step 3.

The computational effort required for considering all possible vertices, i.e., all $N_{\text {in }}$ available interpolation regions, compared to that required for one vertex only is shown in Table I.

\section{Worst Case Design Algorithms}

The steps taken by these algorithms are shown in detail for the two-section transmission-line transformer example given in Section VII (refer to Fig. 10).

\section{Phase 1: Single Interpolation Region}

Step 1: Choose initial values for $\phi^{0}$, $\epsilon$, and $\delta \geqslant \epsilon$.

Step 2: $\bar{\phi} \leftarrow \boldsymbol{\phi}^{0}$.

Step 3: Choose $N$ base points to satisfy (21).

Step 4: Evaluate the constraint functions at these base points.

Step 5: Solve (17) to obtain the coefficients of the interpolating polynomials.

Step 6: Starting with the current $\phi^{\circ}$ and $\epsilon$ solve the nonlinear program WCD for optimal values $\phi^{0 *}$ and $\epsilon^{*}$, employing the constraint approximations defined by Step 5.

Comment: Since values of design constraints as well as their sensitivities at vertices are required in solving WCD, the efficient technique for polynomial evaluation at vertices is used, namely, APE. Obviously, $I_{p}=\{1\}$ for all constraints, since there is only one interpolation region.

Step 7: $\phi^{0} \leftarrow \phi^{0 *}$ and $\epsilon \leftarrow \epsilon^{*}$.

Step 8: If $\left|\phi_{i}^{0}-\bar{\phi}_{i}\right| \leqslant 1.5 \delta_{i}$ for all $i=1,2, \cdots, k$, go to Step 10.

Comment: This tests whether the new norninal point $\phi^{0}$ is close enough to $\bar{\phi}$ to ensure confidence in the accuracy of the approximations.

Step 9: Until $\delta_{i} \geqslant \epsilon_{i}$ for all $i=1,2, \cdots, k$, set $\delta_{i} \leftarrow 4 \delta_{i}$. Go to Step 2.

Comment: Here, we ensure that all the vertices are contained in the interpolation region before repeating Phase 1.

Step 10: Stop if $\boldsymbol{\delta}$ is sufficiently small.

Step 11: $\delta \leftarrow \delta / 4$.

Step 12: If $\delta \geqslant \epsilon$ go to Step 2.

Comment: This check ensures that a single interpolation region is still applicable. If it is, Phase 1 is repeated.

Step 13: Go to Phase 2. 


\section{Phase 2: Multiple Interpolation Regions}

This phase of the worst case design problem is executed if greater accuracy of the solution is required than is possible with the single interpolation region employed in Phase 1. The efficiency will be improved if suitable candidates for active constraints are determined so that not only would fewer interpolations be necessary but also fewer constraints would enter WCD. Step 1 of the present algorithm, therefore, calls for executing Phase 1, and collecting information about candidates for active vertices $I_{\mathrm{av}}$ and corresponding candidates for active constraints $I_{\mathrm{ac}}^{s}, s \in I_{\mathrm{av}}$.

Step 1: Choose $\delta_{\mathrm{ac}}$ as a small positive number and execute Phase 1 to get

$$
\begin{aligned}
& I_{\mathrm{av}} \triangleq\left\{s \mid P_{i}^{s} \leqslant \delta_{\mathrm{ac}}, i \in I_{c}, s \in I_{v}\right\} \\
& I_{\mathrm{ac}}^{s} \triangleq\left\{i \mid P_{i}^{s} \leqslant \delta_{\mathrm{ac}}, i \in I_{c}, s \in I_{v}\right\} .
\end{aligned}
$$

Comment: The set $I_{\mathrm{av}}$ is termed the set of candidates for active vertices. The set $I_{\mathrm{ac}}^{s}$ identifies the corresponding candidates for active constraints associated with the $s$ th vertex.

Step 2: Use (35) to locate centers of interpolation $\bar{\phi}^{l}$ for all $r \in I_{\mathrm{av}}$.

Comment: Note that a subset of all possible interpolation regions is hereby identified because $I_{\mathrm{av}} \subset I_{v}$.

Step 3: For each interpolation region $\bar{R}^{l}$ identified by $\bar{\phi}^{l}$ and $\delta$ :

a) Choose $N$ base points to satisfy (21).

b) $I_{\mathrm{ac}}^{l} \leftarrow \cup_{s \in I_{\mathrm{v}}^{\prime}} I_{\mathrm{acc}}^{s}$.

c) Evaluate $g_{i}$ for all $i \in I_{\mathrm{ac}}^{l}$ at the $N$ base points.

d) Solve (17) to obtain the coefficients of the corresponding polynomials for all $i \in I_{\mathrm{ac}}^{l}$.

Comment: The set $I_{\mathrm{ac}}^{l}$ identifies all the constraints to be evaluated in $\bar{R}^{l}$.

Step 4: Starting with the current $\phi^{0}$ and $\epsilon$ solve the nonlinear program WCD for optimal $\phi^{0 *}$ and $\epsilon^{*}$ employing the constraint approximations defined by Step 3 . Algorithm APE is called for each constraint $i$ to be evaluated by setting $I_{p}(i) \leftarrow\left\{l \mid i \in I_{\text {ac }}^{l}\right\}$.

Comment: Note that the set $I_{v}^{l}$ replaces $I_{v}$ and $I_{\mathrm{ac}}^{l}$ replaces $I_{c}$, thereby reducing the computational effort. Furthermore, $I_{p}(i)$ which becomes $I_{p}$ on entry to APE concentrates evaluations in critical interpolation regions. (See the comment following Step 2.)

Step 5: $\boldsymbol{\phi}^{0} \leftarrow \boldsymbol{\phi}^{0 *}$ and $\boldsymbol{\epsilon} \leftarrow \boldsymbol{\epsilon}^{*}$.

Step 6: $I_{\mathrm{av}} \leftarrow\left\{s \mid P_{i}^{s} \leqslant \delta_{\mathrm{ac}}, i \in I_{\mathrm{ac}}^{\prime}, s \in I_{v}^{\prime}\right\}$ $I_{\mathrm{ac}}^{s} \leftarrow\left\{i \mid P_{i}^{s} \leqslant \delta_{\mathrm{ac}}, i \in I_{\mathrm{ac}}^{l}, s \in I_{v}^{l}\right\}$.

Comment: The set of candidates for active vertices and associated candidates for active constraints is updated by examining all the constraints used during Step 4. Refer to the comment following Step 4.

Step 7: If, for any $s \in I_{\mathrm{av}},\left|\phi_{j}^{s}-\bar{\phi}_{j}^{\prime}\right|>2 \delta_{j}$ for any $j$ go to Step 2.

Step 8: Stop if $\boldsymbol{\delta}$ is sufficiently small.

Step 9: $\boldsymbol{\delta} \leftarrow \delta / 4$.

Step 10: Go to Step 2.

\section{Yield Estimation and Yield Sensitivities}

For a uniform distribution of outcomes inside the tolerance orthotope, computation of hypervolume plays the basic role in yield evaluation. A formula for the nonfeasible hypervolume (hypervolume outside the constraint region but inside the tolerance orthotope) is hereby derived. It is based upon linear cuts of the orthotope.

\section{The Linear Cut and Evaluation of Hypervolume}

Based upon either linearization or intersections (as elaborated on later in this section) of the hypersurface $g(\phi)=0$ with the tolerance orthotope $R_{\epsilon}$, we construct the linear cut

$$
\boldsymbol{q}^{T} \boldsymbol{\phi}-c \geqslant 0
$$

where $\boldsymbol{q}$ is a column vector of $k$ components and $c$ is a scalar. We will derive a general expression for the nonfeasible hypervolume defined by this linear cut and $R_{\epsilon}$, denoted by $V(R)$, where

$$
R=\{\phi \mid g(\phi)<0\} \cap R_{\epsilon} .
$$

Define a reference vertex

$$
\phi^{r}=\phi^{0}+E_{\mu}^{r}
$$

where

$$
\mu_{i}^{r}=-\operatorname{sgn}\left(q_{i}\right), \quad i=1,2, \cdots, k .
$$

The general formula for the hypervolume can be written as

$$
V=\left(\frac{1}{k !} \prod_{j=1}^{k} \alpha_{j}\right)\left(\sum_{s=1}^{2^{k}}(-1)^{\nu^{\prime}}\left(\delta^{s}\right)^{k}\right)
$$

where

$$
\begin{aligned}
& \delta^{s}=\max \left(0,1-\sum_{j=1}^{k} \frac{\epsilon_{j}}{\alpha_{j}}\left|\mu_{j}^{s}-\mu_{j}^{r}\right|\right) \\
& \nu^{s}=\sum_{i=1}^{k}\left|\mu_{i}^{s}-\mu_{i}^{r}\right| / 2
\end{aligned}
$$

and $\alpha_{j}$ is the distance between the intersections of the hyperplane $q^{T} \phi-c=0$ and the reference vertex $\phi^{r}$ along an edge of $R_{\epsilon}$ in the $j$ th direction. It is to be noted that $\delta^{s}$ is positive if and only if the vertex $\phi^{s}$ violates the linear cut (48).

Fig. 6 illustrates the evaluation of hypervolumes for two cases when $k=3$.

\section{Hypervolume Sensitivities}

The hypervolume sensitivities can be expressed as

$$
\begin{aligned}
\frac{\partial V}{\partial \phi_{i}^{0}}=\frac{1}{k !}\left(\sum_{j=1}^{k} \frac{\partial \alpha_{j}}{\partial \phi_{i}^{0}} \prod_{\substack{p=1 \\
p \neq j}}^{k} \alpha_{p}\right) B \\
+A\left(k \sum_{s=1}^{2^{k}}(-1)^{\nu^{s}}\left(\delta^{s}\right)^{k-1} \frac{\partial \delta^{s}}{\partial \phi_{i}^{0}}\right)
\end{aligned}
$$



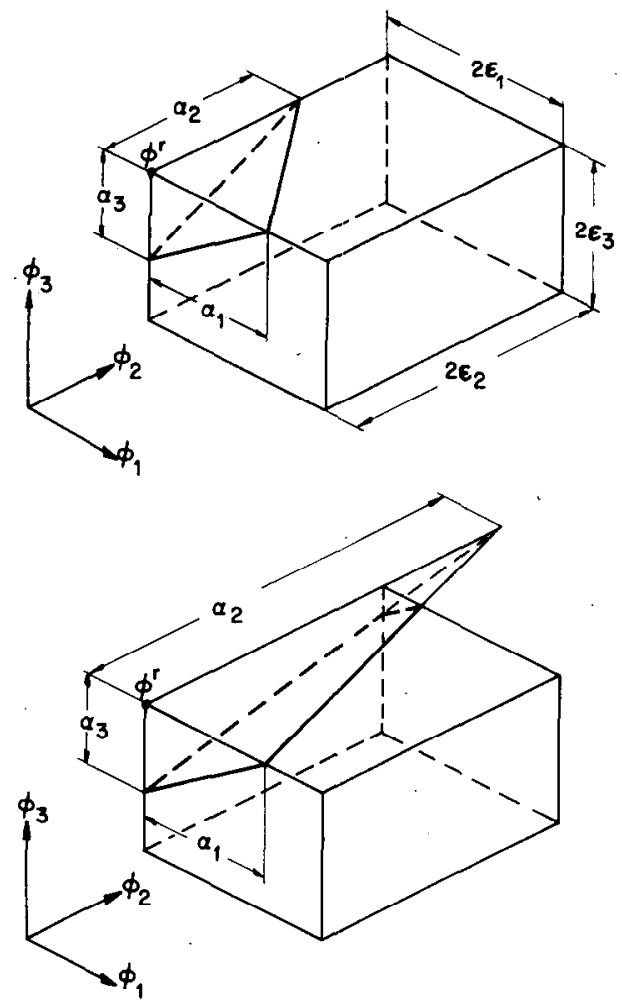

Assuming no overlapping of nonfeasible regions defined by different constraints inside the orthotope $R_{e}$, i.e.,

$$
R_{i} \bigcap_{i \neq j} R_{j}=\varnothing
$$

where

$$
R_{l} \triangleq\left\{\phi \in R_{\epsilon} \mid g_{l}(\phi)<0\right\}
$$

the yield can be expressed as

$$
Y=1-\sum_{l=1}^{m} V\left(R_{l}\right) / V\left(R_{\epsilon}\right) .
$$

Knowing that

$$
V\left(R_{\epsilon}\right)=2^{k} \prod_{j=1}^{k} \epsilon_{j}
$$

the yield sensitivities are given by

$$
\begin{aligned}
\frac{\partial Y}{\partial \phi_{i}^{0}} & =-\sum_{l=1}^{m} \frac{\partial V^{l}}{\partial \phi_{i}^{0}} /\left(2^{k} \prod_{j=1}^{k} \epsilon_{j}\right) \\
\frac{\partial Y}{\partial \epsilon_{i}} & =\left(\frac{1}{\epsilon_{i}} \sum_{l=1}^{m} V^{l}-\sum_{l=1}^{m} \frac{\partial V^{l}}{\partial \epsilon_{i}}\right) /\left(2^{k} \prod_{j=1}^{k} \epsilon_{j}\right)
\end{aligned}
$$

Fig. 6. The nonfeasible volume obtained by a linear cut (a) $V=$ $(1 / 3 !) \alpha_{1} \alpha_{2} \alpha_{3}$. (b) $V=\left((1 / 3 !) \alpha_{1} \alpha_{2} \alpha_{3}\right)\left[1-\left(1-\left(2 \epsilon_{2} / \alpha_{2}\right)\right)^{3}\right]$.

where $V^{l}$ denotes $V\left(R_{l}\right)$. The linear constraints can be used as linear cuts directly. Hence, the nonfeasible hypervolume $V^{l}$ and its sensitivities can be obtained using (52), (55), and (56) for each constraint and where

and

$$
\frac{\partial V}{\partial \epsilon_{i}}=\mu_{i}^{r} \frac{\partial V}{\partial \phi_{i}^{0}}-A\left(\frac{k}{\alpha_{i}} \sum_{s=1}^{2^{k}}(-1)^{\nu^{s}}\left|\mu_{i}^{s}-\mu_{i}^{r}\right|\left(\delta^{s}\right)^{k-1}\right)
$$

where

$$
\begin{aligned}
& A=\frac{1}{k !} \prod_{j=1}^{k} \alpha_{j} \\
& B=\sum_{s=1}^{2^{k}}(-1)^{\nu^{s}}\left(\delta^{s}\right)^{k}
\end{aligned}
$$

and

$$
\frac{\partial \delta^{s}}{\partial \phi_{i}^{0}}= \begin{cases}0, & \text { if } \delta^{s}=0 \\ \sum_{j=1}^{k} \frac{\epsilon_{j}}{\left(\alpha_{j}\right)^{2}}\left|\mu_{j}^{s}-\mu_{j}^{r}\right| \frac{\partial \alpha_{j}}{\partial \phi_{i}^{0}}, & \text { if } \delta^{s}>0 .\end{cases}
$$

It should be mentioned that the hypervolume and its . sensitivities are defined when $\alpha_{i} \rightarrow \infty$ for any $i$, since the limit exists. But, the sensitivities are discontinuous whenever a vertex $\phi^{s}$ satisfies the equation

$$
\boldsymbol{q}^{T} \boldsymbol{\phi}^{s}-c=0 .
$$

The Linear Constraints Case

Let the constraint region be defined by the $m$ linear constraints

$$
g_{l}(\boldsymbol{\phi})=\boldsymbol{\phi}^{T} \boldsymbol{q}^{l}-c^{l} \geqslant 0, \quad l=1,2, \cdots, m .
$$

$$
\begin{aligned}
\alpha_{j}^{l} & =\mu_{j}^{r} g_{l}\left(\phi^{r}\right) / q_{j}^{l} \\
& =\mu_{j}^{r}\left(\sum_{i=1}^{k} q_{i}^{l}\left(\phi_{i}^{0}+\mu_{i}^{r} \epsilon_{i}\right)-c^{l}\right) / q_{j}^{l} \\
\frac{\partial \alpha_{j}^{l}}{\partial \phi_{i}^{0}} & =\mu_{j}^{r} q_{i}^{l} / q_{j}^{l} .
\end{aligned}
$$

If $q_{j}^{l}=0$ we have $\alpha_{j}^{l}=\infty$, however, a limit exists as indicated after (59).

\section{The Quadratic Constraints Case}

Consider a vertex $\phi^{r}$ detected to be active with respect to a quadratic constraint $g_{l}(\phi) \geqslant 0$ after the worst case design process (see Section IV). If the tolerances are allowed to increase slightly beyond their worst case values, intersections between the orthotope edges passing through $\phi^{r}$ and the hypersurface $g_{l}(\phi)=0$ will arise. The number of these intersections is $k$, which is the number of edges passing through $\phi^{r}$, if

$$
\partial g_{l}\left(\phi^{r}\right) / \partial \phi_{j} \neq 0, \quad \text { for all } j \text {. }
$$

In order to find the intersection point along the $j$ th edge, or its extension in the direction $-\mu_{j}^{\prime} e_{j}$, where $e_{j}$ is a unit vector in the $j$ th direction, we express $g_{l}(\phi)=0$ as

$$
\begin{aligned}
\left(\phi_{j}\right)^{2}+2 \phi_{j} \xi_{l}\left(\phi_{1}^{r}, \phi_{2}^{r}, \cdots, \phi_{j-1}^{r}, \phi_{j+1}^{r}, \cdots, \phi_{k}^{r}\right) & \\
& +\eta_{l}\left(\phi_{1}^{r}, \phi_{2}^{r}, \cdots, \phi_{j-1}^{r}, \phi_{j+1}^{r}, \cdots, \phi_{k}^{r}\right)=0
\end{aligned}
$$

where $\xi_{l}$ and $\eta_{l}$ are constant functions and $\phi_{j}$ is the only variable. Hence, the point of intersection is 


$$
\begin{aligned}
&\left(\phi_{1}^{r}, \phi_{2}^{r}, \cdots, \lambda_{j}^{l}, \cdots, \phi_{k}^{r}\right), \text { where } \\
& \lambda_{j}^{l}=-\xi_{l} \pm \sqrt{\xi_{l}^{2}-\eta_{l}}, \quad \mu_{j}^{r}\left(\phi_{j}^{r}-\lambda_{j}^{l}\right)>0
\end{aligned}
$$

is a real root of (71). The condition imposed on the root insures that it is in the direction $-\dot{\mu}_{j}^{r} \boldsymbol{e}_{j}$ with respect to $\boldsymbol{\phi}^{r}$. If both roots lie in this direction, the one closer to $\phi^{r}$ is chosen.

The equation in $\phi$ of the hyperplane, representing the linear cut, which passes through these $k$ points of intersection is

$$
\boldsymbol{\phi}^{T} \boldsymbol{q}^{l}-c^{l}=\operatorname{det}\left[\begin{array}{ccccc}
\phi_{1} & \phi_{2} & \cdots & \phi_{k} & 1 \\
\lambda_{1}^{l} & \phi_{2}^{r} & \cdots & \phi_{k}^{r} & 1 \\
\phi_{1}^{r} & \lambda_{2}^{l} & \cdots & \phi_{k}^{r} & 1 \\
\vdots & & & & \\
\cdot & & & & \\
\phi_{1}^{r} & \phi_{2}^{r} & \cdots & \lambda_{k}^{l} & 1
\end{array}\right]=0
$$

and $\boldsymbol{\phi}^{r}$ is a reference vertex for this cut.

The yield sensitivities are calculated according to the gradients of the $k$ intersections.

$\frac{\partial \lambda_{j}^{\prime}}{\partial \phi_{i}}=-\frac{\partial \xi_{l}}{\partial \phi_{i}} \pm \frac{1}{2 \sqrt{\xi_{l}^{2}-n_{l}}}\left(2 \xi_{l} \frac{\partial \xi_{l}}{\partial \phi_{i}}-\frac{\partial \eta_{l}}{\partial \phi_{i}}\right), \quad i \neq j$

$\frac{\partial \lambda_{i}^{\prime}}{\partial \phi_{i}}=0$

Thus if $\alpha_{j}^{l}$ is the distance from the vertex $\phi^{r}$ to the point of intersection with the $l$ th constraint along the orthotope edge in the $j$ th direction, then

$$
\begin{aligned}
\alpha_{j}^{l} & =\mu_{j}^{r}\left(\phi_{j}^{r}-\lambda_{j}^{l}\right) \\
\frac{\partial \alpha_{j}^{l}}{\partial \phi_{i}^{0}} & =-\mu_{j}^{r} \frac{\partial \lambda_{j}^{l}}{\partial \phi_{i}}, \quad i \neq j \\
\frac{\partial \alpha_{j}^{l}}{\partial \phi_{j}^{0}} & =\mu_{j}^{r} .
\end{aligned}
$$

Equations (76), (77), and (78) are substituted directly into formulas (52), (55), and (56), whichever is relevant. Yield and its sensitivities are also obtained from (64), (66), and (67).

\section{Overlapping Constraints}

We discuss in this section an approach which is directed at solving some of the problems arising from constraints overlapping within the tolerance region. Since the analytical formulas for yield and yield sensitivities assume nonoverlapping linear cuts (see (62)), methods to avoid describing the boundary of the constraint region by overlapping cuts are required.

A single function of the least $p$ th type [9] can be used to describe the boundary of the feasible region if the boundary is defined, as is usual, by more than one con- straint. The least $p$ th function is given by

$$
G\left(g_{i}(\phi), I_{c}, p\right)= \begin{cases}0, & M=0 \\ M\left[\sum_{i \in J}\left(g_{i}(\phi) / M\right)^{q}\right]^{1 / q}, & M \neq 0\end{cases}
$$

where

$$
\begin{aligned}
M & =\min _{i \in I_{c}} g_{i}(\phi) \\
J & = \begin{cases}\left\{i \mid g_{i}(\phi)<0, i \in I_{c}\right\}, & \text { if } M<0 \\
I_{c}, & \text { if } M>0\end{cases} \\
q & =-p \operatorname{sgn} M
\end{aligned}
$$

and $p$ is given to be greater than 1 .

The constraint $G \geqslant 0$ exactly describes the boundary of the constraint region $R_{c}$.

In order to define a linear cut based on $G$, we can either linearize $G$ at an appropriate point or use intersections of the hypersurface $G=0$ with the orthotope edges. A possible implementation is suggested in the appropriate steps of the following algorithms.

\section{Algorithm FOR Yifid I.ess than 100 PERCENT}

It is assumed that Phase 1 and Phase 2 of the worst case design algorithm have been suitably executed. Information has, therefore, been gathered relating to active vertices $I_{\mathrm{av}}$, associated active constraints $I_{\mathrm{ac}}^{s}$ at the $s$ th vertex and also polynomial approximations $P_{i}(\phi)$ corresponding to the (generally) nonlinear $g_{i}(\phi)$. The least $p$ th function $G_{s}\left(P_{i}(\phi), I_{\mathrm{ac}}^{s}, p\right), s \in I_{\mathrm{av}}$, can be formulated according to the notation introduced by (79) and is associated with the $s$ th vertex.

Note also that optimal values $\phi^{0 *}$ and $\epsilon^{*}$ are known for worst case design. See Fig. 7.

Step 1: For $\kappa_{i}>1$ set $\epsilon_{i} \leftarrow \kappa_{i} \epsilon_{i}^{*}, i=1,2, \cdots, k$.

Comment: This initializes the yield to be less than $10 \mathrm{C}$ percent. The $\kappa_{i}$ are chosen such that all active constraints are violated, as indicated by Fig. 7(b).

Step 2: $\phi^{0} \leftarrow \boldsymbol{\phi}^{0 *}$.

Step 3: Solve the nonlinear program YNP for optima values $\phi^{0 *}$ and $\epsilon^{*}$ employing algorithm YAN (which follows) for evaluating yield and yield sensitivities.

\section{Algorithm for Dynamic Yield Analysis (YAN)}

This algorithm is called for each evaluation of yield anc its sensitivities as required during optimization.

Step 1: $S \leftarrow\left\{s \mid s \in I_{\mathrm{av}}, G_{s}\left(P_{i}\left(\phi^{s}\right), I_{\mathrm{ac}}^{s}, p\right)<0\right\}$.

Comment: $S$ is a working set of indices of referenc vertices $(1,2$, and 3 in Fig. 7(a)). We consider only thos vertices which currently violate the design constraints fo the nonfeasible hypervolume evaluation $(1,2$, and 3 is Fig. 7(b)).

Step 2: $V \leftarrow 0, V_{\phi_{i}} \leftarrow 0$, and $V_{\epsilon_{i}} \leftarrow 0, i=1,2, \cdots, k$.

Comment: $V, V_{\phi}$, and $V_{\epsilon}$ are to be updated to store th total nonfeasible hypervolume and its sensitivities wit. respect to $\phi^{0}$ and $\epsilon$, respectively. 


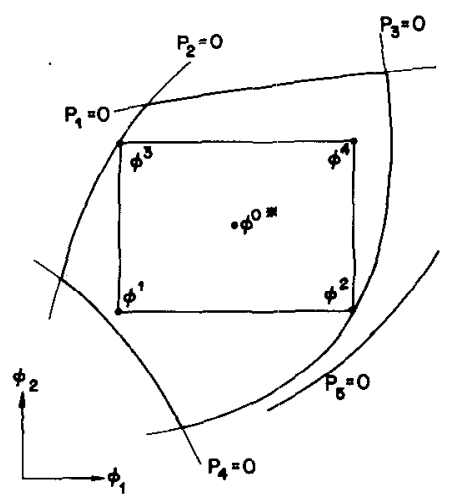

(a)

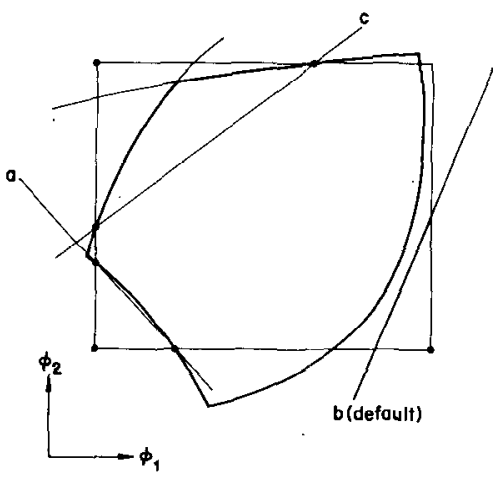

(b)

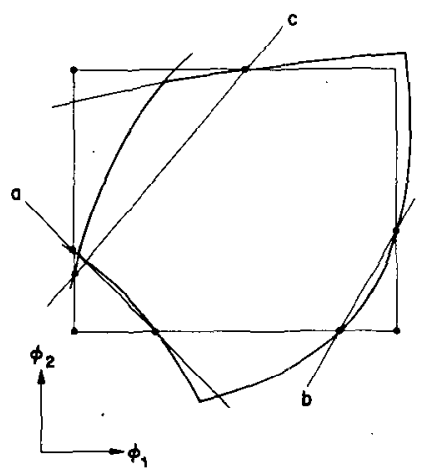

(c)

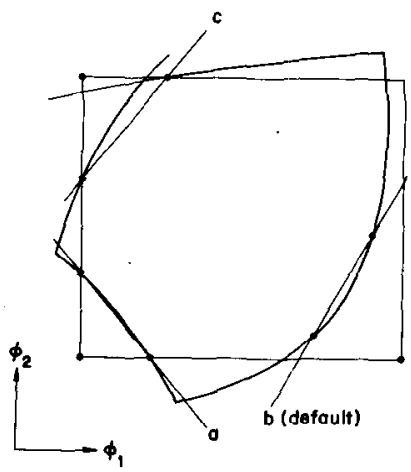

(d)

ig. 7. Examples of yield determination. (a) Quadratic constraint ap proximations and a worst case design. $I_{\mathrm{av}}=\{1,2,3\}, I_{\mathrm{ac}}^{1}=\{4\}, I_{\mathrm{ac}}^{2}=$ $\{3,5\}, I_{\text {ac }}^{3}=\{1,2\}$. Notice that $P_{1}$ and $P_{2}$ are overlapping and $P_{5}$ is a redundant constraint. (b) Linear cuts arising after setting $\epsilon_{i} \leftarrow \kappa_{i} \epsilon_{i}^{*}$. Cuts $a$ and $c$ are based upon intersections, while cut $b$ is based upon linearization. (c) A typical situation which may result during optimization, in which all cuts are based on intersections. (d) A situation which may arise during optimization. The linear cut $b$ is not updated since less than $k$ intersections exist, remaining as in (c).
Step 3: $r \leftarrow \min _{s \in S} s$.

Comment: This ordering process selects the index $r$ corresponding to the reference vertex to be considered.

Step 4: For $j=1,2, \cdots, k$, execute Steps 5 and 6.

Comment: In this loop we consider the edges of the orthotope passing through $\phi^{r}$ as indicated in Steps 5 and 6.

Step 5: Find $\lambda_{j}^{l}$, for all $l \in I_{\text {ac }}^{r}$, using (72).

Step 6: If $\lambda_{j}^{l}$ is undefined for any $l \in I_{\mathrm{ac}}^{r}$, go to Step 14.

Comment: The hypersurface $G_{r}=0$ has an. intersection with an orthotope edge if $P_{l}$ has an intersection with the edge for all $l \in I_{\mathrm{ac}}^{r}$. We go to Step 14 if such intersections are not found for all $k$ edges.

Step 7: If $I_{\mathrm{ac}}^{r}$ contains more than one element, go to Step 10.

Comment: In case $I_{\mathrm{ac}}^{r}$ contains one element only, $l$ say, there is no need to consider $G_{r}$, since $G_{r}=P_{l}$.

Step 8: Find $\alpha_{j}^{r}$ and $\partial \alpha_{j}^{r} / \partial \phi_{i}^{0}, i, j=1,2, \cdots, k$, where $I_{\mathrm{ac}}^{r}=\{l\}$, using (76), (77), and (78).

Comment: Notice that we will identify the cut by index of the reference vertex $r$ rather than using $l$.

Step 9: Go to Step 12.

Step 10: $\alpha_{j}^{r} \leftarrow \max _{l \in I_{a c}^{r}} \alpha_{j}^{l}, j=1,2, \cdots, k$, where $\alpha_{j}^{l}$ is obtained by (76).

Comment: The furthest intersection, from $\phi^{r}$, among the intersections of $P_{l}=0, l \in I_{\text {ac }}^{r}$, corresponds to the intersection of the hypersurface $G_{r}=0$.

Step 11: Find $\partial \alpha_{j}^{r} / \partial \phi_{i}^{0}, i, j=1,2, \cdots, k$, using (77) and (78).

Step 12: Set $\boldsymbol{q}^{r}$ and $c^{r}$ for the $r$ th linear cut according to (73).

Comment: In general, the explicit formulation of the linear cut is not necessary since information about $\alpha_{j}^{r}$ is the only requirement for hypervolume calculation. But this cut will be used later in the process as a clefault if less than $k$ intersections are obtained (Fig. 7(d)).

Step 13: Go to Step 17.

Step 14: If this is not the first yield evaluation, go to Step 16.

$$
\begin{aligned}
\text { Step 15: } & q^{r} \leftarrow \nabla G_{r}\left(P_{l}^{r}, I_{\mathrm{ac}}^{r}, p\right) \\
& c^{r} \leftarrow\left(\phi^{r}\right)^{T} \boldsymbol{q}^{r}-G_{r}\left(P_{l}^{r}, I_{\mathrm{ac}}^{r} p\right) .
\end{aligned}
$$

Comment: Initially if less than $k$ intersections exist, linearization at the vertex $\boldsymbol{\phi}^{\prime}$ is used to provide a default cut. Cut $b$ in Fig. 7(b) is an example.

Step 16: Find $\alpha_{j}^{r}$ and $\partial \alpha_{j}^{r} / \partial \phi_{i}^{0}$ using (68) and (69).

Comment: No updating of the $r$ th cut is performed.

Step 17: $V \leftarrow V+V^{r}$

$$
\begin{gathered}
V_{\phi_{i}} \leftarrow V_{\phi_{i}}+V_{\phi_{i}}^{r}, \quad i=1,2, \cdots, k \\
V_{c_{i}} \leftarrow V_{\epsilon_{i}}+V_{c_{i}}^{r}, \quad i=1,2, \cdots, k
\end{gathered}
$$

where $V^{r}$ is given by (52), $V_{\phi_{i}}^{r}$ by (55) and $V_{\epsilon_{i}}^{r}$ by (56), respectively.

Step 18: $S \leftarrow S \backslash\{r\}$.

Comment: The index $r$, already exploited, is removed from the working set $S$.

Step 19: If $S \neq \varnothing$ go to Step 3 .

Comment: This step checks if all reference vertices have been considered. 
TABLE II

Worst Case Design of THE Two-Section 10: 1 Quarter-Wave TRANSFORMER

\begin{tabular}{cccccccc}
\hline $\begin{array}{c}\text { Cost } \\
\text { Function }\end{array}$ & $z_{1}^{0}$ & $z_{2}^{0}$ & $\begin{array}{c}\varepsilon_{1} / z_{1}^{0} \\
(\%)\end{array}$ & $\begin{array}{c}\varepsilon_{2} / z_{2}^{0} \\
(\%)\end{array}$ & $\delta$ & N.O.F.E. & $\begin{array}{l}\text { CDC } \\
\text { Time } \\
(\mathrm{sec})\end{array}$ \\
\hline \multirow{2}{*}{$\mathrm{C}_{1}$} & 2.5637 & 5.5048 & 14.678 & 9.007 & 0.4 & 18 & 7.2 \\
& 2.5234 & 5.4379 & 14.988 & 9.081 & 0.1 & 24 & 9.5 \\
\multirow{2}{*}{$\mathrm{C}_{2}$} & 2.1515 & 4.7350 & 12.715 & 12.697 & 0.4 & 12 & 2.5 \\
& 2.1494 & 4.7305 & 12.687 & 12.700 & 0.1 & 18 & 3.0 \\
\hline
\end{tabular}

Starting values $z_{1}^{0}-2.2361, z_{2}^{0}=4.4721, c_{1}=0.2$ and $\varepsilon_{2}=0.4$

Frequency points used $0.5,0.6, \ldots, 1.5 \mathrm{GHz}$

objective cost functions $C_{1}=\frac{1}{\varepsilon_{1}}+\frac{1}{\varepsilon_{2}}, C_{2}=\frac{z_{1}^{0}}{\varepsilon_{1}}+\frac{z_{2}^{0}}{\varepsilon_{2}}$

Reflection coefficient specification $|\rho| \leq 0.55$

*N.O.F.E. denotes the number of function evaluations

Step 20: $\quad Y \leftarrow 1-V / V\left(R_{\epsilon}\right)$

$$
\begin{aligned}
& \partial Y / \partial \phi_{i}^{0} \leftarrow-V_{\phi_{i}} / V\left(R_{\epsilon}\right), \quad i=1,2, \cdots, k \\
& \partial Y / \partial \epsilon_{i} \leftarrow\left[V / \epsilon_{i}-V_{\epsilon}\right] / V\left(R_{\epsilon}\right), \quad i=1,2, \cdots, k
\end{aligned}
$$

where $V\left(R_{\epsilon}\right)$ is given by (65).

\section{EXAMPLes}

\section{Two-Section Transmission-Line Transformer}

Consider the two-section 10:1 quarter-wave lossless transmission-line transformer used by Bandler et al. [1]. The specifications and results of the worst case tolerance optimization problem of the characteristic impedances $Z_{1}$ and $Z_{2}$ over 100 -percent bandwidth are shown in Table II for two different objective functions. The constraint region and the resulting optimum solutions in two cases are shown in Fig. 8 and Fig. 9. An equal value of $\delta_{1}$ and $\delta_{2}$ was used. The figures show the interpolation regions and the resulting approximations for the constraint boundary. The results obtained are contrasted with the results obtained in [1]. Furthermore, the steps taken by the worst case design algorithm using the objective function $C_{1}$, shown in Table II, are detailed in Fig. 10.

Subsequently, the approximations obtained at the two active vertices for the worst case problem having the objective function $C_{1}$, shown in Table II and Fig. 8, were used for yield optimization. This problem is denoted $P 0$. A rough estimate of $\delta$ used for stopping Phase 2 was obtained in the following way. For a yield constraint

\section{$Y \geqslant 90$ percent}

the nonfeasible hypervolume (it is area in this example) is given approximately by

$$
A \simeq(1-0.9)\left(2 \epsilon_{1}\right)\left(2 \epsilon_{2}\right) \text {. }
$$

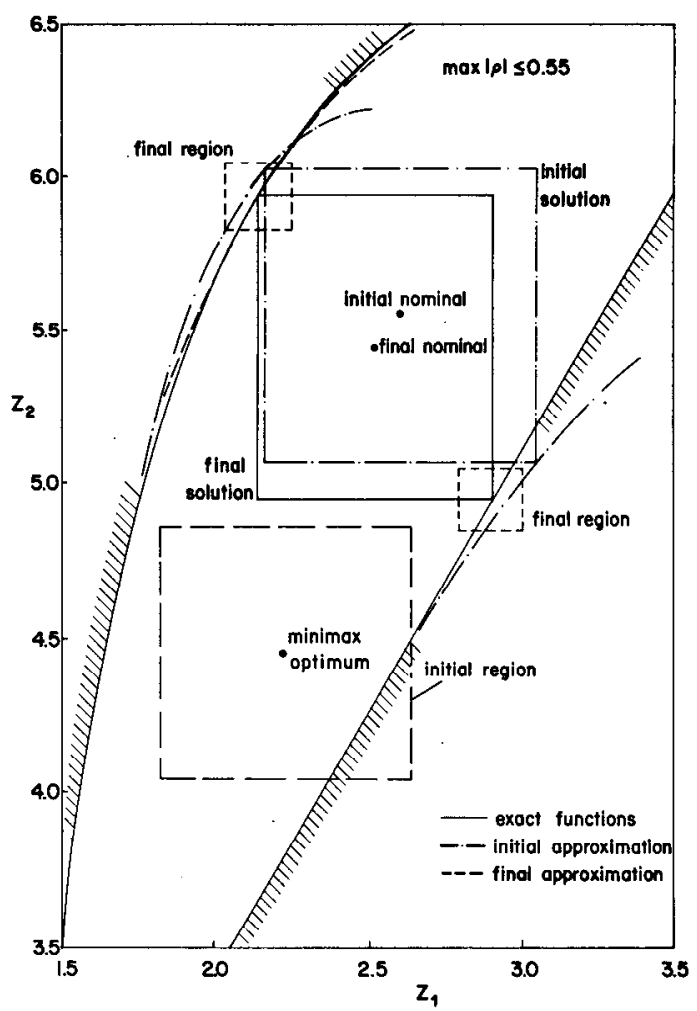

Fig. 8. Minimization of $1 / \epsilon_{1}+1 / \epsilon_{2}$ for the two-section transformer.

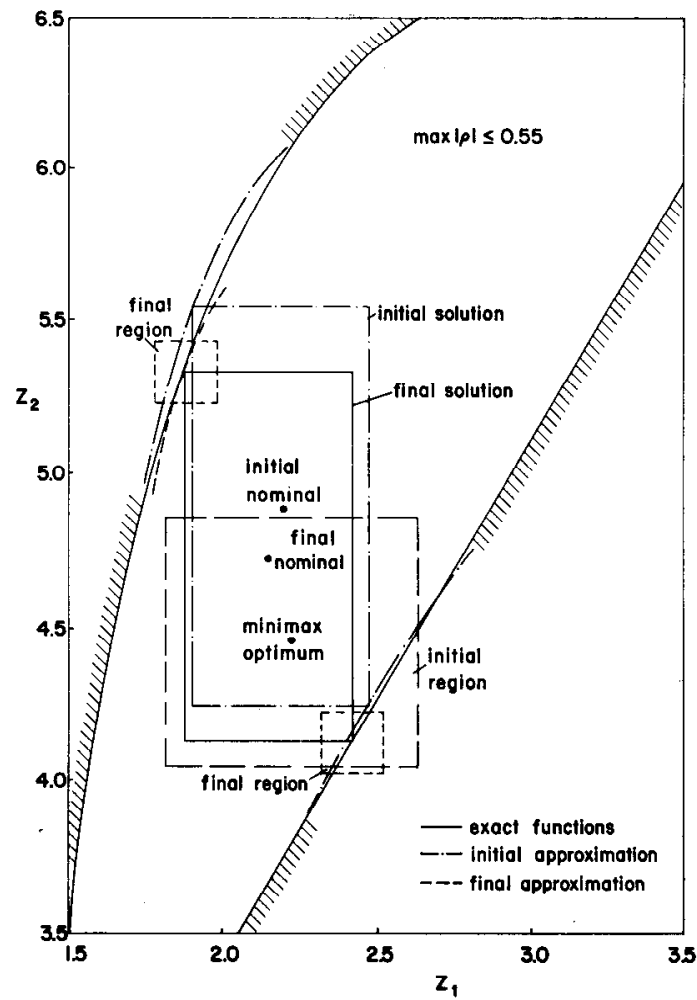

Fig. 9. Minimization of $Z_{1}^{0} / \epsilon_{1}+Z_{2}^{0} / \epsilon_{2}$ for the two-section trans-
former. 


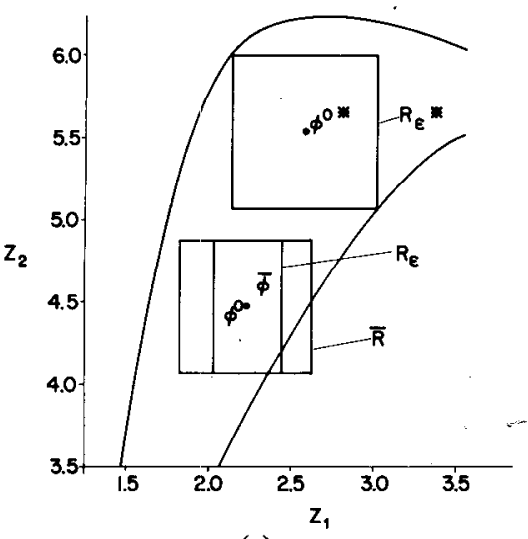

(a)

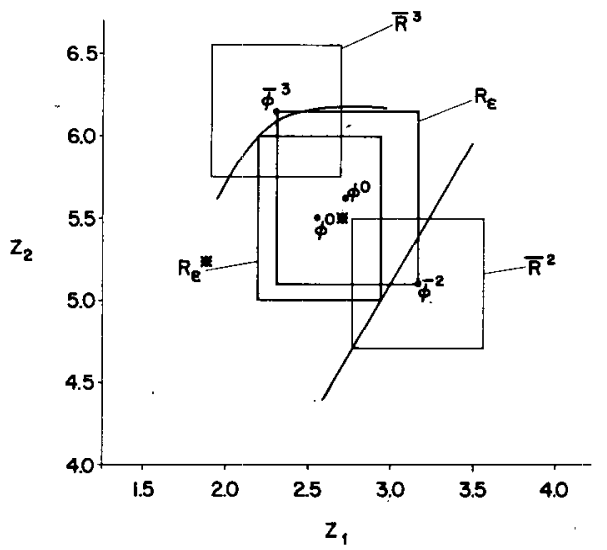

(c)

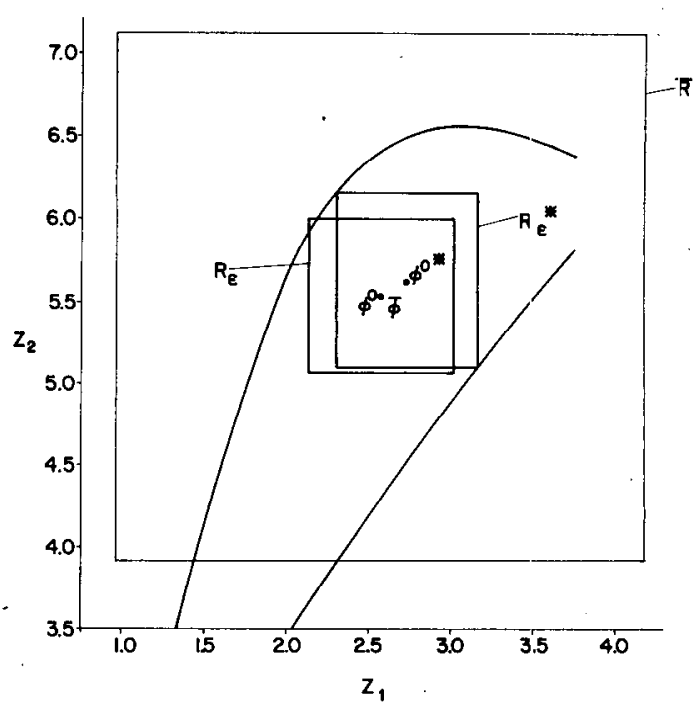

(b)

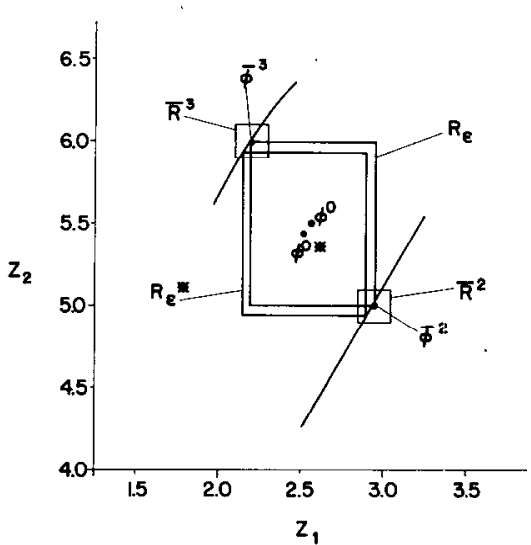

(d)

Fig. 10. Details for the problem of Fig. 8. (a) Initial interpolation region $\bar{R}$, tolerance region $R_{\varepsilon}$ and the approximations to the boundary of the constraint region. $R_{\varepsilon}^{*}$ is the optimum tolerance region using Phase 1 of the worst case design algorithm. (b) Enlarged interpolation region $\bar{R}$ and starting with the previous optimum $R_{\varepsilon}$ we arrive at $R_{\varepsilon}^{*}$. (c) Reducing the interpolation region size and switching to Phase 2 of the algorithm. $I_{\mathrm{ac}}^{2}=\{$ frequency point $1.0 \mathrm{GHz}\}$ and $I_{\mathrm{ac}}^{3}=$ frequency points 0.5 and $1.5 \mathrm{GHz}$. (d) Further reduction in interpolation region size resulting in the final optimum solution $R_{\varepsilon}^{*}$.

The area cut off by each constraint is

$$
A^{\prime} \simeq \frac{1}{2} A
$$

But, assuming equal intersections $\alpha=\alpha_{1}=\alpha_{2}$

$$
A^{\prime}=\frac{1}{2} \alpha^{2}
$$

Hence

$$
\alpha \simeq \sqrt{0.1\left(2 \epsilon_{1}\right)\left(2 \epsilon_{2}\right)}=0.27
$$

where $\epsilon_{1}$ and $\epsilon_{2}$ are the worst case absolute tolerances. The approximation with $\delta=0.1$ was used for solving problems

$$
\begin{gathered}
P 1\left\{\begin{array}{l}
\text { minimize } 1 / \epsilon_{1}+1 / \epsilon_{2} \\
\text { subject to } \\
Y \geqslant 90 \text { percent }
\end{array}\right. \\
P 2 \text { minimize }\left(1 / \epsilon_{1}+1 / \epsilon_{2}\right) / Y
\end{gathered}
$$

assuming a uniform distribution of outcomes between tolerance extremes.

The optimum solutions for $P 1$ and $P 2$ are shown in Table III and contrasted with the worst case solution $P 0$ in Fig. 11. The program FLNLP2 [15] was used for solving the resulting nonlinear programming problem. Since a convex constraint region appears in this problem, the values of yield obtained are lower bounds for the true yields.

\section{Three-Component LC Low-pass Filter}

A normalized three-component low-pass ladder network, terminated with equal load and source resistances of $1 \Omega$ is shown in Fig. 12. The circuit was considered for worst case design by Bandler, Liu, and Chen [1]. Although this filter is symmetric a three-dimensional approximation was required in order to perform the yield technique described before. 
TABLE III

Yield Determination AND Optimization OF THE TWO-SeCtion 10: 1 QuARTER-WAVE TRANSFORMER

\begin{tabular}{|c|c|c|c|c|c|c|c|c|}
\hline Problem & $\mathrm{z}_{1}^{0}$ & $\mathrm{z}_{2}^{0}$ & $\begin{array}{c}E_{1} / z_{1}^{0} \\
(\%)\end{array}$ & $\underset{\varepsilon_{2} / z_{2}^{0}}{(\%)}$ & Objective & $\begin{array}{c}\text { Yield } \\
\text { (\%) }\end{array}$ & N.O.Y.E.*** & $\begin{array}{l}\text { cuc } \\
\text { Time } \\
\text { (sec) }\end{array}$ \\
\hline $\mathrm{P}_{1}$ * & 2.5273 & 5.3998 & 21.09 & 13.51 & 3.2465 & 90.0 & 45 & 0.6 \\
\hline $\mathrm{P} 2 * *$ & 2.5290 & 5.1513 & 31.44 & 22.13 & 3.2597 & 65.5 & 15 & 0.3 \\
\hline \multicolumn{9}{|c|}{$\begin{array}{l}\text { * Minimize } 1 / \varepsilon_{1}+1 / \varepsilon_{2} \text { subject to yield } \leq 90^{\circ} \\
\text { ** Minimize }\left(1 / \varepsilon_{1}+1 / \varepsilon_{2}\right) / Y \\
\text { *** N.O.Y.E. denotes the number of yield evaluations } \\
\text { Starting point for both P1 and P2 is } z_{1}^{0}=2.5234, z_{2}^{0}=5.4379 \text { (worst case nomina1) } \\
\text { and } \kappa_{1}=\kappa_{2}=1.4\end{array}$} \\
\hline
\end{tabular}

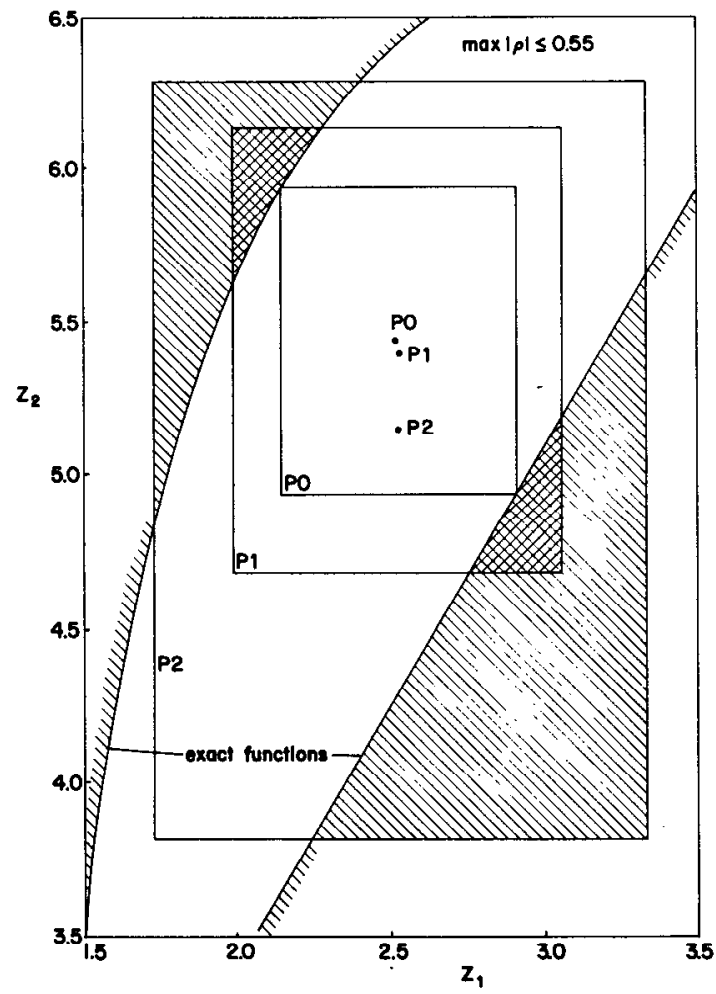

Fig. 11. The optimum tolerance regions and nominal values for the worst case, 90-percent yield and optimum yield designs.

Using an equal step size $\delta$ for all components, a worst case design was first obtained with final $\delta=0.01$. The base points used are given by (21) with

$$
\boldsymbol{B}=\left[\begin{array}{rrr}
0.5 & -0.5 & 1.0 \\
-0.5 & 0.5 & 1.0 \\
0.8 & 0.8 & 1.0
\end{array}\right]
$$

consistent with the vector of components

$$
\phi=\left[\begin{array}{l}
L_{1} \\
L_{2} \\
C
\end{array}\right]
$$

The specifications and the objective function are given in Table IV. The convergence of the quadratic approxima-

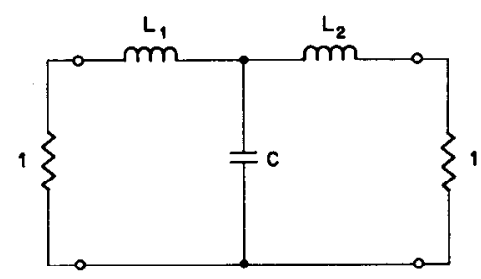

Fig. 12. The circuit for the $L C$ filter.

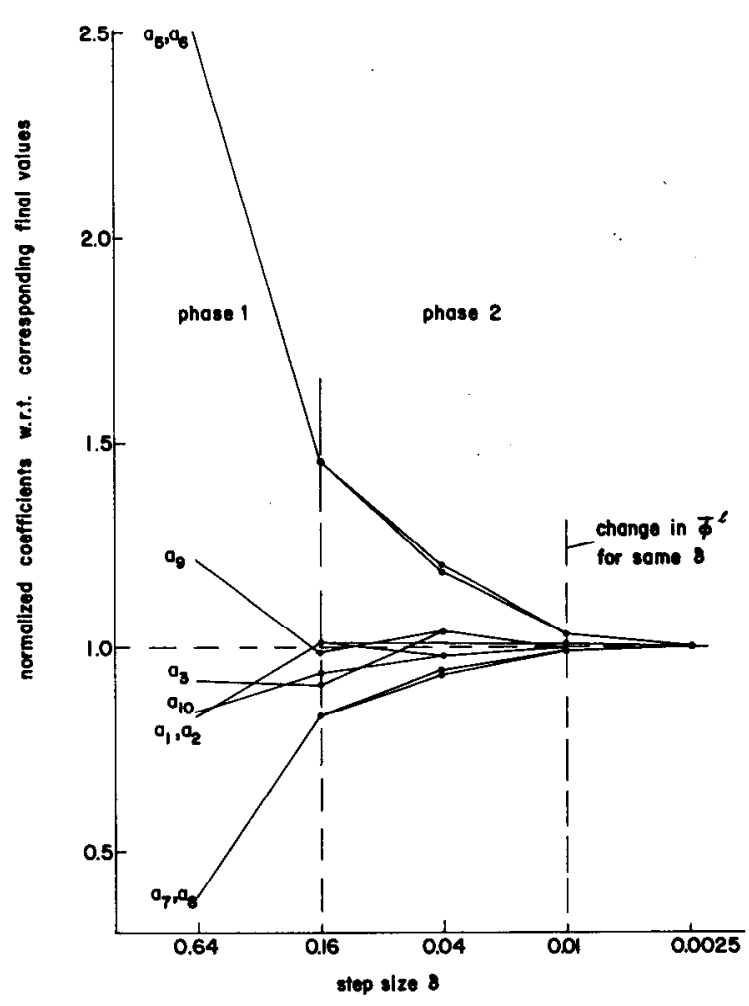

Fig. 13. Convergence for the $L C$ filter of the quadratic approximation to the insertion loss constraint at $2.5 \mathrm{rad} / \mathrm{s}$.

tion coefficients as the step size $\delta$ is reduced is shown in Fig. 13 for the insertion loss constraint at the frequency point $2.5 \mathrm{rad} / \mathrm{s}$. The coefficient $a_{4}$ is not shown in the figure. Its value is close to zero and hence the normalized 
TABLE IV

Worst Case and Constrained Yield Results of the LC Low-Pass Filter

\begin{tabular}{ccccccccc}
\hline $\begin{array}{c}\text { Yield } \\
\left(\frac{\%}{6}\right)\end{array}$ & $\mathrm{L}_{1}^{0}$ & $\mathrm{~L}_{2}^{0}$ & $\mathrm{C}^{0}$ & $\begin{array}{c}\varepsilon_{1} / \mathrm{L}_{1}^{0} \\
(\%)\end{array}$ & $\begin{array}{c}\varepsilon_{2} / \mathrm{L}_{2}^{0} \\
(\%)\end{array}$ & $\begin{array}{c}\varepsilon_{\mathrm{C}^{\circ}} / \mathrm{C}^{0} \\
(\%)\end{array}$ & N.0.Y,E.* & $\begin{array}{l}\text { CDC } \\
\text { Time } \\
(\mathrm{sec})\end{array}$ \\
\hline 100 & 1.999 & 1.998 & 0.9058 & 9.88 & 9.89 & 7.60 & - & 1.9 \\
96 & 1.997 & 1.997 & 0.9033 & 11.23 & 11.23 & 12.46 & 38 & 1.0 \\
\hline
\end{tabular}

* N.O.Y.E. denotes the number of yield evaluations

Frequency points used are $0.45,0.5,0.55,1.0$ in the passband and 2.5 in the stopband

Objective cost function is $\mathrm{L}_{1}^{0} / \varepsilon_{1}+\mathrm{L}_{2}^{0} / \varepsilon_{2}+\mathrm{C}^{0} / \varepsilon_{\mathrm{C}}$

Insertion loss specification is $\leq 1.5 \mathrm{~dB}$ in the passband and $\geq 25 \mathrm{~dB}$ in the stopband

Starting point for the worst casc design problem is $L_{1}^{0}=L_{2}^{0}=2.0, C^{0}=1.0$ and

$\varepsilon_{1} / L_{1}^{0}=\varepsilon_{2} / L_{2}^{0}=\varepsilon_{C} / C^{0}=10 \%$

Starting point for the $96 \%$ yield design problem is the optimal worst case nominal with

$\kappa_{1}=\kappa_{2}=1.06$ and $\kappa_{3}=1.45$

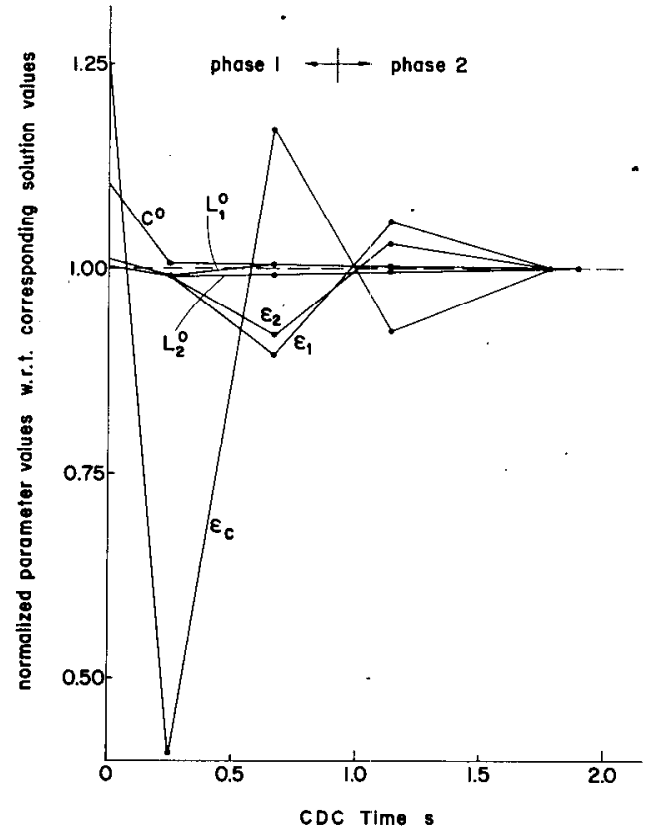

Fig. 14. Parameter values for the worst case design of the $L C$ filter as a function of execution time.

value is highly oscillatory. Corresponding parameter values are shown in Fig. 14 as a function of execution time. At the worst case optimum, given in Table IV, the active frequency point constraints are $0.55,1.0$, and $2.5 \mathrm{rad} / \mathrm{s}$.

Now, consider the problem given by

$$
\begin{aligned}
& \text { minimize } L_{1}^{0} / \epsilon_{1}+L_{2}^{0} / \epsilon_{2}+C^{0} / \epsilon_{C} \\
& \text { subject to } \\
& \quad Y \geqslant 96 \text { percent. }
\end{aligned}
$$

The quadratic approximation with $\delta=0.04$, which was used in this problem, is shown in Table $\mathrm{V}$ after and before averaging symmetric coefficients. The diagonal elements. of the Hessian matrix, as defined by the coefficients of the

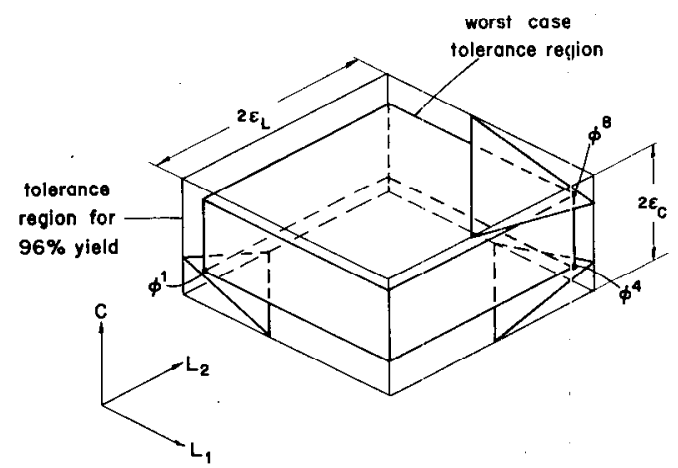

Fig. 15. The tolerance regions for the worst case design and the 96-percent yield for the $L C$ filter. The linear cuts shown are based on the intersections of the active quadratic constraints approximations with edges of the tolerance region for 96-percent yield case. $I_{\mathrm{ac}}^{1}=$ (frequency point $2.5 \mathrm{rad} / \mathrm{s}\}, I_{\mathrm{ac}}^{4}=\{$ frequency point $0.55 \mathrm{rad} / \mathrm{s}$ \}, and $I_{\mathrm{ac}}^{8}=\{$ frequency point $1.0 \mathrm{rad} / \mathrm{s}\}$.

approximating polynomial, suggest a one-dimensionally convex constraint region. Symmetry between $L_{1}$ and $L_{2}$ was used to reduce computation in finding the values and the gradients of the intersections between the orthotope edges and the quadratic constraints. The results are shown in Table IV and in Fig. 15. The tolerance for: the capacitor $\epsilon_{C}$ was approximately doubled, with respect to its value for the worst case design, by allowing the yield to drop to 96 percent. (A Monte Carlo analysis at the solution indicated 96.6-percent yield by both the exact constraints and by the approximate ones.)

\section{Two-Section Waveguide Transformer}

The two-section waveguide transformer, investigated for a minimax (equal-ripple) response by Bandler [16] was selected to perform a tolerance assignment. The general configuration of such a structure is illustrated in Fig. 16. A design specification of a reflection coefficient of 0.05 over $500-\mathrm{MHz}$ bandwidth centered at $6.175 \mathrm{GHz}$ was chosen. Table VI shows the dimensions of the input and output waveguides and the widths of the-tworsections. 
TABLE V

CoffFicients of the Quadratic Approximation ARound ACtive Vertices

\begin{tabular}{|c|c|c|c|c|c|c|c|c|c|c|c|}
\hline $\begin{array}{l}\text { Freq. } \\
\text { point }\end{array}$ & State & $\mathrm{L}_{1}^{2}$ & $\mathrm{~L}_{2}^{2}$ & $c^{2}$ & $\mathrm{~L}_{1} \mathrm{~L}_{2}$ & $\mathrm{~L}_{1} \mathrm{C}$ & $\mathrm{L}_{2} \mathrm{C}$ & $L_{1}$ & $\mathbf{L}_{2}$ & $\mathrm{C}$, & - \\
\hline \multirow{2}{*}{0.55} & before & -0.06847 & -0.06847 & -0.57056 & .33010 & 0.92247 & 0.93855 & -1.67845 & -1.69182 & -0.46249 & 3.83750 \\
\hline & after & -0.06847 & -0.06847 & -0.57056 & .33010 & 0.93051 & 0.93051 & -1.68513 & -1.68513 & -0.46249 & 3.83750 \\
\hline 1.00 & after & -1.14445 & -1.14445 & -9.98122 & .21439 & -8.23326 & -8.23326 & 10.36637 & 10.36637 & 44.18607 & -33.86206 \\
\hline \multirow{2}{*}{2.50} & before & -1.38601 & -1.12228 & -9.90167 & .39487 & -0.92910 & -0.94732 & 10.19142 & 10.32736 & 32.94001 & -46.93184 \\
\hline & after & -1.40414 & -1.40414 & -9.90167 & .39487 & -0.93821 & -0.93821 & 10.25939 & 10.25939 & 32.94001 & -46.93184 \\
\hline
\end{tabular}

Coefficients of the quadratic approximations obtained at active vertices with a step $\delta=0.04$. The table shows the coefricients obtained by the algorithm and the coefficients used for yield determination after averaging symmetric coefficients.

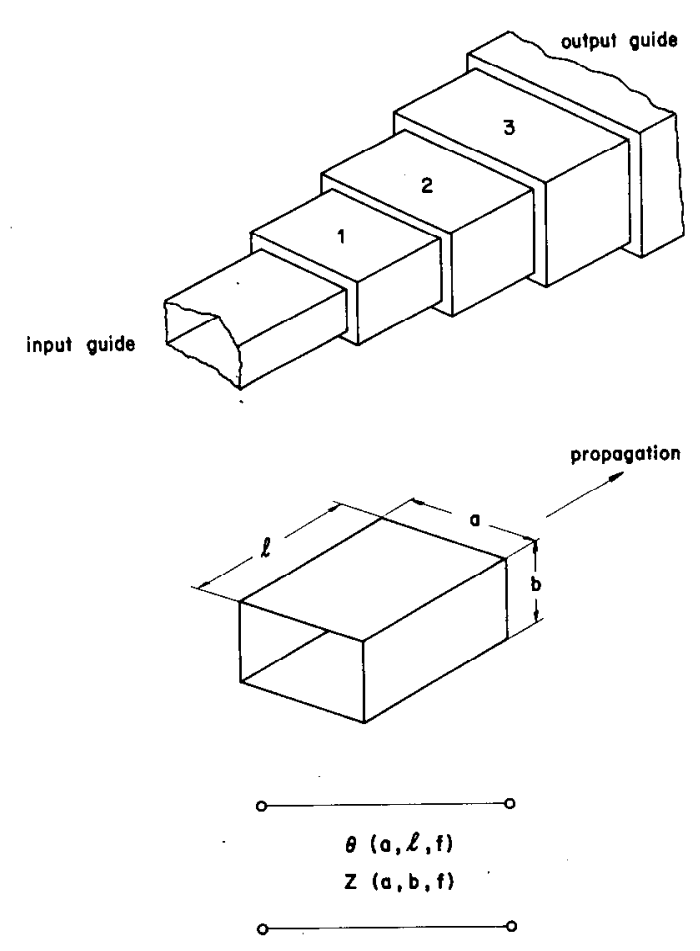

Fig. 16. Illustrations of an inhomogeneous waveguide transformer.

The program developed by Bandler and Macdonald [17] is used to obtain the reflection coefficient. No sensitivities are provided by this program. An equal ábsolute tolerance $\epsilon$ is assumed for the heights and the lengths of the two sections. The assumption seems reasonable if they are machined in the same manner.

The objective is to maximize the absolute tolerance $\epsilon$. The optimum nominal point and associated tolerance, given in Table VII, were obtained by the worst case design algorithm presented in Section IV. The program FLOPT4 [18] was used for solving the nonlinear program:

subject to maximize $\epsilon$

$$
R_{v} \subset R_{c}
$$

TABLE VI

Fixed PARAMETERS AND SPECIFICATIONS For THE TWO-SECTION WAVEGUIDE TRANSFORMER

\begin{tabular}{lllc}
\hline Description & $\begin{array}{l}\text { Width } \\
(\mathrm{cm})\end{array}$ & $\begin{array}{c}\text { Height } \\
\text { (cm) }\end{array}$ & $\begin{array}{c}\text { Length } \\
(\mathrm{cm})\end{array}$ \\
\hline Input guide & 3.48488 & 0.508 & $\infty$ \\
First section & 3.6 & variable & variable \\
Second section & 3.8 & variable & variable \\
Output guide & 4.0386 & 2.0193 & $\infty$ \\
\hline
\end{tabular}

Frequency points used $5.925,6.175,6.425 \mathrm{GHz}$

Reflection coefficient specification $|\rho| \leq 0.05$

Minimax solution (no tolerances) $|\rho|=0.00443$

TABLE VII

Results CONTRAsting THE TOLERANCED SOLUTION AND THE MINIMAX SOLUTION WITH NO TOLERANCES FOR THE TWO-Section Waveguide Transformer

\begin{tabular}{lcccc}
\hline \hline Description & $\begin{array}{c}\mathrm{b}_{1} \\
(\mathrm{~cm})\end{array}$ & $\begin{array}{c}\mathrm{b}_{2} \\
(\mathrm{~cm})\end{array}$ & $\begin{array}{c}\ell_{1} \\
(\mathrm{~cm})\end{array}$ & $\begin{array}{c}\ell_{2} \\
(\mathrm{~cm})\end{array}$ \\
\hline $\begin{array}{l}\text { Toleranced } \\
\text { optimum }\end{array}$ & 0.72812 & 1.42432 & .1 .55409 & 1.51153 \\
$\begin{array}{l}\text { Minimax } \\
\text { optimum }\end{array}$ & 0.71315 & 1.39661 & 1.56044 & 1.51621 \\
\hline
\end{tabular}

Equal absolute value of tolerance $=0.02013 \mathrm{~cm}$

Number of complete response evaluations $=45$

$\operatorname{CDC}$ time (approximation and optimization) $=33 \mathrm{~s}$

A tolerance of $0.02013 \mathrm{~cm}$ was obtained. The number of actual response evaluations to reach the optimum starting from the minimax optimum (no tolerances) is shown in Table VII. The execution time shown includes both approximation and optimization times. 


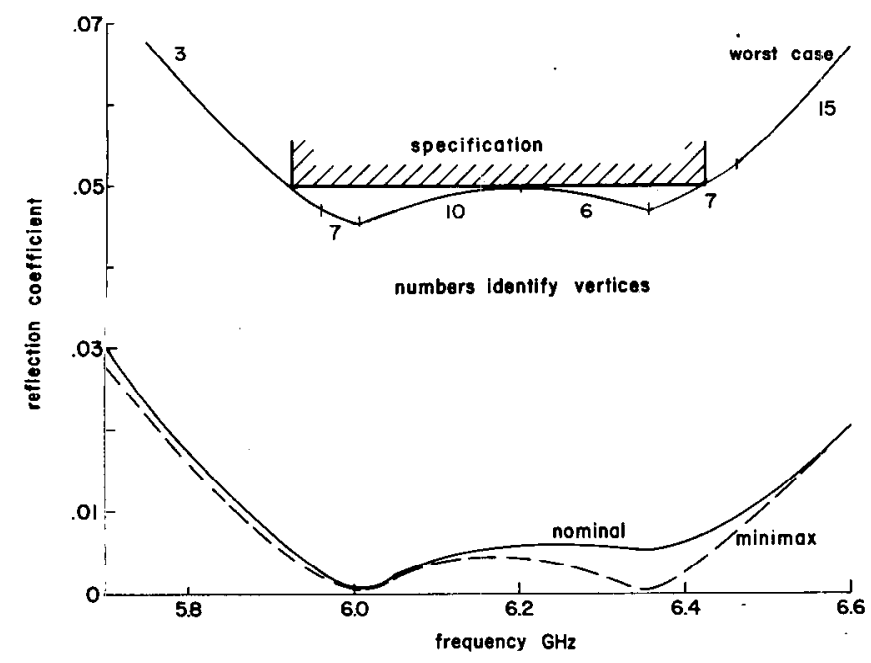

Fig. 17. Nominal, minimax and upper envelope of worst case responses for the two-section waveguide transformer.

The minimax, nominal and the upper envelope of worst case responses are shown in Fig. 17. The numbering scheme of the vertices is that given by (38) with the parameter vector

$$
\boldsymbol{\phi}=\left[\begin{array}{l}
b_{1} \\
b_{2} \\
l_{1} \\
l_{2}
\end{array}\right] .
$$

Vertices which fall within the worst case upper envelope are not indicated in Fig. 17. It was observed, however, that vertices $2,6,10$, and 14 are either active or almost active with respect to the reflection coefficient constraint at band center. Furthermore, vertices 3, 7, 11, and 15 are either active or almost active near the band extremes. Hence, when $b_{1}$ is at its positive extreme while $b_{2}$ is at its negative extreme, the frequency point at the center of the band is more likely to be violated. The edges of the band are critical frequency points when $b_{1}$ is at its negative extreme while $b_{2}$ is at its positive extreme.

Retaining the approximations obtained by the worst case design procedure subsequently facilitates inexpensive Monte Carlo analyses. Hence, different statistical distributions of outcomes may be assumed and estimates of corresponding yields obtained. Assuming $\epsilon=0.03 \mathrm{~cm}$, for example, while keeping the worst case nominal obtained, uniformly distributed Monte Carlo analyses were conducted with the approximation and with the actual functions: for 500 points, yields of 88 and 89 percent, respectively, are predicted. The approximation produces results 12 times faster.

\section{Three-Section Waveguide Transformer}

The three-section transformer with ideal junctions for which a minimax optimum was obtained by Bandler [16] is considered for tolerance assignment. Specifications and dimensions of input and output waveguides are given in Table VIII.
TABLE VIII

Fixed Parameters and SPECIFICATIONS fOr the Three-Section WAVEGUIDE TRANSFORMER

\begin{tabular}{lccc}
\hline Description & $\begin{array}{c}\text { Width } \\
(\mathrm{cm})\end{array}$ & $\begin{array}{c}\text { Height } \\
(\mathrm{cm})\end{array}$ & $\begin{array}{c}\text { Length } \\
(\mathrm{cm})\end{array}$ \\
\hline Input guide & 3.48488 & 0.762 & $\infty$ \\
First section & 3.30581 & variable & variable \\
Second section & 3.12674 & variable & variable \\
Third section & 2.94767 & variable & variable \\
Output guide & 2.76860 & 1.60325 & $\infty$ \\
\hline
\end{tabular}

Frequency points used $5.7,6.1,6.45,6.8,7.2 \mathrm{GHz}$

Reflection coefficient specification $|\rho|<0.050$ (nonideal junctions)

Minimax solution (no tolerances) $|\rho|=0.017$ (ideal junctions)

TABLE IX

RESUlts CONTRASTING THE TOLERANCED SOLUTION AND THE MiNIMAX SOLUTION WITH No TOLERANCES IOR THE THREe-Section WaVEguid:e Transformar

\begin{tabular}{lccccccc}
\hline Description & $\begin{array}{c}\mathrm{b}_{1} \\
(\mathrm{~cm})\end{array}$ & $\begin{array}{c}\mathrm{b}_{2} \\
(\mathrm{~cm})\end{array}$ & $\begin{array}{c}\mathrm{b}_{3} \\
(\mathrm{~cm})\end{array}$ & $\begin{array}{c}\ell_{1} \\
(\mathrm{~cm})\end{array}$ & $\begin{array}{c}\ell_{2} \\
(\mathrm{~cm})\end{array}$ & $\begin{array}{c}\ell_{3} \\
(\mathrm{~cm})\end{array}$ \\
\hline $\begin{array}{l}\text { Toleranced } \\
\text { optimum }\end{array}$ & 0.91034 & 1.36526 & 1.70189 & 1.45242 & 1.53875 & 1.63253 \\
$\begin{array}{l}\text { Minimax } \\
\text { optimum }\end{array}$ & 0.90318 & 1.37093 & 1.73609 & 1.54879 & 1.58375 & 1.64590 \\
\hline
\end{tabular}

Equal absolute value of tolerance $=0.01383 \mathrm{~cm}$

Number of complete response evaluations $=56$

CDC time (approximation and optimization) $=167 \mathrm{~s}$

Nonideal junctions were assumed and the widths of the three sections were fixed for convenience, so that the step changes are equal from one section to the next. An equal tolerance in the heights and lengths of the three sections was maximized for the reason already given.

Starting at the minimax optimum with equal steps of 0.02 for the interpolation region the results shown in Table IX were obtained. The program FLOPT4 [18] was used for solving the nonlinear programming problem formulated for the worst case design. Fig. 18 shows the upper envelope of worst case responses as well as the nominal design response. Although the envelope shows one vertex which is active at the lower frequency edge of the band, several other adjacent vertices, which restricted the increase in tolerance, are almost active. This appears to explain the fact that the envelope is substantially lower than the specification at other frequencies.

To verify the accuracy of the approximation an equal tolerance of $0.02 \mathrm{~cm}$ was assumed around the worst case nominal design and 500 uniformly distributed points were generated. The yield for the approximations was 96.4 percent and for the actual functions 96.0 percent. A twelve-fold improvement in execution time was again observed. 


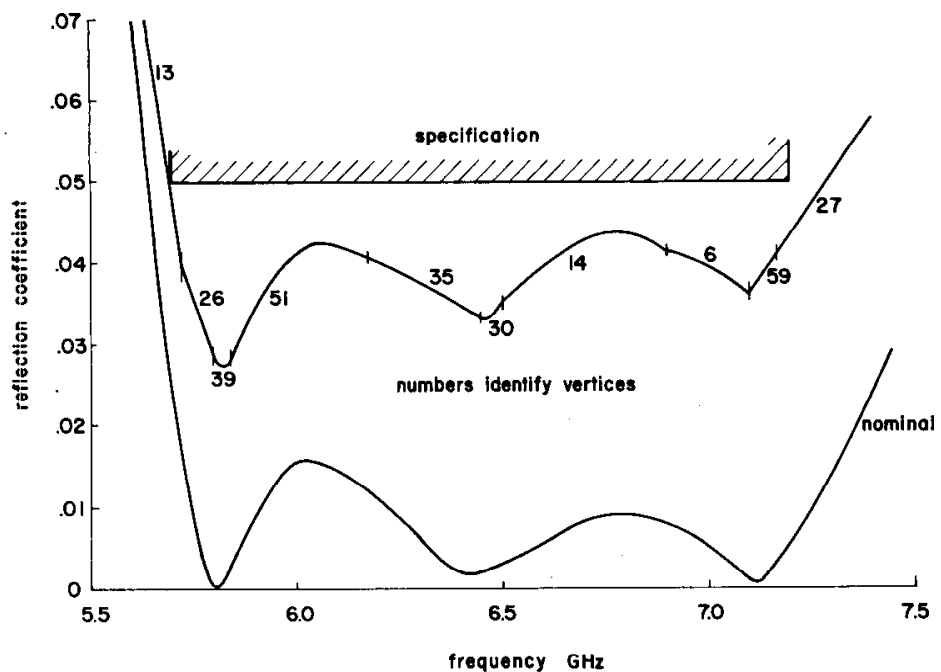

Fig. 18. Nominal and upper envelope of worst case responses for the three-section waveguide transformer.

\section{ConCLusions}

A design centering technique based upon low-order multidimensional approximation and nonlinear programming is presented. The technique bridges the gap between available analysis programs, which may or may not be efficiently written and probably do not supply derivative information, and the advancing art of optimal centering, tolerancing and tuning. Efficient gradient methods, which are essential in such general design problems, can be usefully employed through the use of readily differentiable formulas and approximations.

In order to contrast various design centering techniques which rely on approximations, we point out that the method of Pinel and Roberts [3] and that of Karafin [4] are based upon truncated Taylor series expansions. Hence, not only sensitivities are required but also the validity of such an approximation for relatively large tolerances is uncertain. The simplicial approximation technique [6] does not require sensitivities, but the convexity assumption used is much more restrictive than the - one-dimensional convexity assumption used in the present technique. Moreover, the approximation developed for the constraint region by Director and Hachtel [6] does not contain sensitivity information which allows the designer to check the effect of slightly relaxing some constraints. However, in the present technique, since there exists at least one quadratic approximation to each constraint it is possible to remove a constraint completely or slightly perturb its value (by changing the constant term in the quadratic approximation) to study such an effect on the design.

As expected, the design centering technique presented here facilitates subsequent inexpensive Monte Carlo analysis. For circuits which are expensive to analyze, such as switching circuits, this technique may be cheaper even for a single yield analysis using the Monte Carlo method in conjunction with the approximation. It is difficult to contrast our approach with the simplicial approximation ap- proach from the point of view of Monte Carlo analysis. The fact that the simplicial approximation approach develops a relatively large number of linear constraints $\left(2^{k}+n k\right.$, where $k$ is the number of design parameters and $n$ is the number of iterations required) while we develop quadratic constraints of the order of the number of actual constraints makes it hard to compare.

In addition, the quadratic approximations devcloped can be used for the new yield estimation and optimization technique developed. The yield estimation technique can also be used by itself if a reasonable worst case design is already known. The linear cuts may be obtained by linearizing active constraints at either associated active vertices or at the nominal point [19]. The technique can be extended to general nonlinear constraints. The efficient technique for calculation of the function and gradients at the different vertices (APE) may then be implemented with a suitable large-change sensitivity algorithm.

Yield estimation for other statistical distributions, different from the uniform distribution, can be done by regionalizing the space and associating a uniform distribution with each region [19].

\section{APPENDIX \\ Preservation of One-Dimensional Convexity}

As described in Section II, one-dimensional convexity is the property which makes the vertices candidates for the worst case. Hence, it is essential to preserve this property in the approximating polynomial $P(\phi)$ if it already exists in the exact function $g(\phi)$.

The following theorem indicates how to choose the base points in order to preserve one-dimensional convexity.

\section{Theorem}

If there exist three distinct base points $\phi^{1}, \phi^{2}$, and $\phi^{3}$ in the $i$ th direction, i.e.,

$$
\boldsymbol{\phi}^{j}=\boldsymbol{\phi}^{1}+c_{j} \boldsymbol{e}_{i}
$$

where $c_{j}, j=2,3$, are scalars and $e_{i}$ is the unit vector in the $i$ th direction, then the interpolating polynomial $P(\phi)$ is one-dimensionally convex/concave in the $i$ th variable if the interpolated function $g(\phi)$ is so.

Proof: Assume that $P(\phi)$ is not one-dimensionally convex/concave, i.e.,

$$
P\left(\lambda \boldsymbol{\phi}^{a}+(1-\lambda) \boldsymbol{\phi}^{b}\right) \gtrless \lambda P\left(\phi^{a}\right)+(1-\lambda) P\left(\phi^{b}\right), \quad 0<\lambda<1
$$

where

$$
\phi^{b}=\phi^{a}+c e_{i}
$$

and where $c$ is a scalar. Hence

$$
P\left(\phi^{a}+(1-\lambda) c \boldsymbol{e}_{i}\right) \gtrless \lambda P\left(\phi^{a}\right)+(1-\lambda) P\left(\phi^{a}+c \boldsymbol{e}_{i}\right) .
$$

Expanding $P\left(\phi^{a}+(1-\lambda) c \boldsymbol{e}_{i}\right)$ and $P\left(\phi^{a}+c \boldsymbol{e}_{i}\right)$ as Taylor series and knowing that $P(\phi)$ is a quadratic polynomial, 
we have

$$
\begin{aligned}
P\left(\phi^{a}\right)+(1-\lambda) c \boldsymbol{e}_{i}^{T} \nabla P\left(\phi^{a}\right)+\frac{1}{2}(1-\lambda)^{2} c^{2} \boldsymbol{e}_{i}^{T} \boldsymbol{H} \boldsymbol{e}_{i} \\
\gtrless P\left(\phi^{a}\right)+(1-\lambda) c \boldsymbol{e}_{i}^{T} \nabla P\left(\phi^{a}\right)+\frac{1}{2}(1-\lambda) c^{2} \boldsymbol{e}_{i}^{T} \boldsymbol{H} \boldsymbol{e}_{i} .
\end{aligned}
$$

Thus

$$
(1-\lambda)^{2} e_{i}^{T} H e_{i} \gtrless(1-\lambda) e_{i}^{T} H e_{i}
$$

but since $0<(1-\lambda)<1$, hence

$$
\boldsymbol{e}_{i}^{T} \mathrm{He}_{i} \lessgtr 0 .
$$

Without any loss of generality we can number the three base points such that

$$
\boldsymbol{\phi}^{3}=\gamma \boldsymbol{\phi}^{1}+(1-\gamma) \boldsymbol{\phi}^{2}, \quad 0<\gamma<1 .
$$

and

$$
\phi^{2}=\phi^{1}+\beta e_{i}
$$

where $\beta$ is a scalar. Then

$$
\begin{aligned}
P\left(\phi^{3}\right)= & P\left(\gamma \phi^{1}+(1-\gamma) \phi^{2}\right) \\
= & P\left(\phi^{1}+(1-\gamma) \beta \boldsymbol{e}_{i}\right) \\
= & P\left(\phi^{1}\right)+(1-\gamma) \beta \boldsymbol{e}_{i}^{T} \nabla P\left(\phi^{1}\right)+\frac{1}{2}(1-\gamma)^{2} \beta^{2} \boldsymbol{e}_{i}{ }^{T} \boldsymbol{H} \boldsymbol{e}_{i} \\
= & \gamma P\left(\phi^{1}\right)+(1-\gamma)\left[P\left(\phi^{1}\right)+\beta \boldsymbol{e}_{i}^{T} \nabla P\left(\phi^{1}\right)+\frac{1}{2} \beta^{2} \boldsymbol{e}_{i}{ }^{T} \boldsymbol{H} \boldsymbol{e}_{i}\right] \\
& -\frac{1}{2}(1-\gamma) \beta^{2} \boldsymbol{e}_{i}^{T} \boldsymbol{H e}_{i}+\frac{1}{2}(1-\gamma)^{2} \beta^{2} \boldsymbol{e}_{i}^{T} \boldsymbol{H} \boldsymbol{e}_{i} \\
= & \gamma P\left(\phi^{1}\right)+(1-\gamma) P\left(\phi^{2}\right)-\frac{1}{2} \gamma(1-\gamma) \beta^{2} \boldsymbol{e}_{i}^{T} \boldsymbol{H} \boldsymbol{e}_{i} .
\end{aligned}
$$

But, using (A7)

$$
P\left(\phi^{3}\right) \gtrless \gamma P\left(\phi^{1}\right)+(1-\gamma) P\left(\phi^{2}\right)
$$

and since $g=P$ at the base points, then

$$
g\left(\boldsymbol{\phi}^{3}\right) \gtrless \gamma g\left(\boldsymbol{\phi}^{1}\right)+(1-\gamma) g\left(\boldsymbol{\phi}^{2}\right)
$$

which contradicts the fact that $g(\phi)$ is one-dimensionally convex/concave in the $i$ th variable. Hence, the assumption (A2) is never true.

Q.E.D.

\section{Corollary}

A quadratic polynomial is one-dimensionally convex/ concave if and only if all of the diagonal elements of its Hessian matrix are nonnegative/nonpositive.

The proof follows since inequality (A7) is never true.

The previous corollary allows an easy check on one-dimensional convexity of any quadratic function. In addition, the choice of base points as given in (21) satisfies the requirement of locating three base points in each direction.

\section{ACKNOWLEDGMENT}

The authors thank R. Biernacki, K. Madsen, M. R. M. Rizk, and $\mathrm{H}$. Tromp for suggesting some improvements in our presentation.

\section{REFERENCES}

[1] J. W. Bandler, P. C. Liu, and J. H. K. Chen, "Worst case network tolerance optimization," IEEE Trans. Microwave Theory Tech., vol. MTT-23, pp. 630-641, Aug. 1975.

[2] J. W. Bandler, P. C. Liu, and H. Tromp, "A nonlinear programming approach to optimal design centering, tolerancing and tuning," IEEE Trans. Circuits Syst., vol. CAS-23, pp. 155-165, Mar. 1976.

[3] J. F. Pinel and K. A. Roberts, "Tolerance assignment in linear networks using nonlinear programming," IEEE Trans. Circuit Theory, vol. CT-19, pp. 475-479, Sept. 1972.

[4] B. J. Karafin, "The optimum assignment of component tolerances for electrical networks," Bell Syst. Tech. J., vol. 50, pp. 1225-1242, Apr. 1971.

[5] N. Elias, "New statistical methods for assigning clevice tolerances," in Proc. 1975 IEEE Symp. Circuits Syst., Newton, MA, pp. 329332, Apr. 1975.

[6] S. W. Director and G. D. Hachtel, "The simplicial approximation approach to design centering and tolerance assignment," in Proc. 1976 IEEE Symp. Circuits Syst., Munich, Germany, pp. 706-709, Apr. 1976.

[7] J. W. Bandler, "Optimization of design tolerances using nonlinear programming," J. Optimization Theory and Appl., vol. 14, pp. 99-114, July 1974.

[8] J. W. Bandler and P. C. Liu, "Some implications of biquadratic functions in the tolerance problem," IEEE Trans. Circuits Syst. vol. CAS-22, pp. 385-390, May 1975.

[9] J. W. Bandler and C. Charalambous, "Practical least $p$ th optimization of networks," IEEE Trans. Microwave Theory Tech., vol. MTT-20, pp. 834-840, Dec. 1972.

[10] H. S. M. Coxeter, Regular Polytopes, 2nd ed., New York: MacMillan, 1963, chap. 7 .

[11] R. K. Brayton, A. J. Hoffman, and T. R. Scott, "A theorem of inverses of convex sets of real matrices with application to the worst case dc problem," IEEE Trans. Circuits Syst., vol. CAS-23, pp. 409-415, Aug. 1977.

[12] S. L. Sobolev, "On the interpolation of functions of $n$ variables" (transl.), Sov. Math. Dokl., vol. 2, pp. 343-346, 1961.

[13] H. C. Thacher, Jr., and W. E. Milne, "Interpolation in several variables," SIAM J., vol. 8, pp. 33-42, 1960.

[14] H. C. Thacher, Jr., "Generalization of concepts related to linear dependence," SIAM J., vol. 6, pp. 288-299, 1959.

[15] W. Y. Chu, "Extrapolation in least $p$ th approximation and nonlinear programming," Faculty of Engineering, McMaster University, Hamilton, Canada, Rep. SOC-71, Dec. 1974.

[16] J. W. Bandler, "Computer optimization of inhomogeneous waveguide transformers," IEEE Trans. Microwave Theory Tech., vol. MTT-17, pp. 563-571, Aug. 1969.

[17] J. W. Bandler and P. A. Macdonald, "Response program for an inhomogeneous cascade of rectangular waveguicles," IEEE Trans. Microwave Theory Tech., vol. MTT-17, pp. 646-649, Aug. 1969.

[18] J. W. Bandler and D. Sinha, "FLOPT4-A program for least $p$ th optimization with extrapolation to minimax solutions," Faculty of Engineering, McMaster University, Hamilton, Canada, Rep. SOC151, Jan. 1977

[19] H. L. Abdel-Malek and J. W. Bandler, "Yield estimation for efficient design centering assuming arbitrary statistical distributions," in Conf. Computer-Aided Design of Electronic and Microwave Circuits and Systems, Hull, England, pp. 66-71, July 1977, also to be published in Int. J. Circuit Theory and Applications.

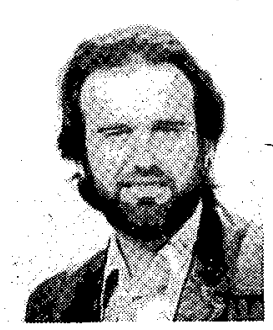

John W. Bandler (S'66-M'66-SM'74-F'78) was born in Jerusalem, Palestine, on November 9, 1941. He studied at Imperial College of Science and Technology, London, England, from 19601966. He received the B.Sc.(Eng.), Ph.D., and D.Sc.(Eng.) degrees from the University of London, London, England, in 1963, 1967, and 1976, respectively.

He joined Mullard Research Laboratories, Redhill, Surrey, England in 1966. From 1967 to 1969 he was a Postdoctorate Fellow and Sessional Lecturer at the University of Manitoba, Winnipeg, Canada. He became Assistant Professor in 1969, Associate Professor in 1971, Pro- 
fessor in 1974 and Chairman of the Department in 1978 in the Department of Electrical Engineering at McMaster University, Hamilton, Canada. He has been Coordinator of the Research Group on Simulation, Optimization and Control since 1973. During part of the year 1975-1976 he was a Visiting Professor at the Technion-Israel Institute of Technology, Haifa, Israel, and the University of Bologna, Bologna, Italy. He is a contributor to Modern Filter Theory and Design (G. C. Temes and S. K. Mitra, Eds., Wiley-Interscience, 1973). He is author or coauthor of over 130 papers, four of which appear in Computer-Aided Filter Design (G. Szentirmai, Ed., IEEE Press, 1973), and one in Microwave Integrated Circuits (J. Frey, Ed., Artech House, 1975). Dr. Bandler was an Associate Editor of the IEEE Transactions on Microwave Theory and Techniques from 1969-1974. He was Guest Editor of the IEEE Transactions on Microwave Theory and Techniques Special Issue on Computer-Oriented Microwave Practices (March 1974).

Dr. Bandler is a member of the Institute of Electrical Engineers (Great Britain) and a member of the Association of Professional Engineers of the Province of Ontario (Canada).

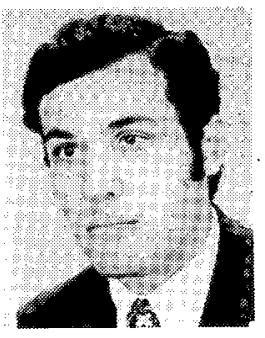

Hany L. Abdel-Malek (S'75) was born in Cairo, Egypt, on January 1, 1949. He received B.Sc. degrees in electrical engineering and in mathematics (both with Honors) from Cairo University, Cairo, Egypt, in 1970 and 1972, respectively, and the Ph.D. degree in electrical engineering from McMaster University, Hamilton, Canada, in 1977.

From 1970 to 1974 he served as an Instructor at the Department of Engineering Physics and Mathematics, Cairo University. He was also a Teaching Assistant at the American University, Cairo, from 1973 to 1974. He was with the Department of Electrical Engineering, McMaster University, Hamilton, Canada from 1974 to 1978. In 1977 he was awarded a Postdoctoral Fellowship by the National Research Council of Canada. His main research interests are in the areas of circuit theory, computer-aided circuit and system design, and optimization techniques. Dr. Abdel-Malek is a member of the Syndicate of Engineers (Egypt).

\title{
Cascade Synthesis of a Class of Multivariable Positive Real Functions
}

\author{
M. O. AHMAD, STUDENT MEMBER, IEEE, HARNATHA C. REDDY, MEMBER, IEEE, \\ V. RAMACHANDRAN, SENIOR MEMBER, IEEE, AND M. N. S. SWAMY, SENIOR MEMBER, IEEE
}

\begin{abstract}
Necessary and sufficient conditions are developed for the realization of a multivariable positive real function (MPRF) as the driving-point function of an extracted single-variable lossless two-port with a termination whose driving-point function is an MPRF in the rest of the variables as the load. Conditions are also obtained for the case when an extraction of a lossless two-port is possible in either of the two variables $p_{i}$ or $p_{j}$.
\end{abstract}

\section{INTRODUCTION}

T N RECENT YEARS, considerable interest has been shown in the study of multivariable positive real functions (MPRF's) because of their applications in the theory and design of electrical networks. In particular, cascade realizations of MPRF's have immediate applications in the design of microwave filters and multidimensional digital filters. Shirakawa et al. [1] have given necessary and sufficient conditions on an MPRF whereby extraction of

Manuscript received June 30, 1977. This work was supported by the National Research Council of Canada under Grant A-7279.

M. O. Ahmad, V. Ramachandran, and M. N. S. Swamy are with the Department of Electrical Engineering, Concordia University, Montreal, P.Q., Canada.

H. C. Reddy was with the Department of Electrical Engineering, Concordia University, Montreal, P.Q., Canada. He is now with the Department of Technology and Business Studies, State University College, Buffalo, NY 14222 . a lowpass ladder with all of its transmission zeros at infinity is possible. More recently, some work [2], [3] has been reported on cascade extraction of a reciprocal section with Darlington-type realization.

In this paper, some new results on the cascade realization of multivariable positive real functions are obtained. The necessary and sufficient conditions for an $m$-variable positive real function (PRF) to be realizable as the driving-point impedance of a lossless two-port in one of the variables, with a termination of an $(m-1)$-variable PRF, are given. Augmentation with surplus factors as in the single-variable Darlington synthesis is not possible here. Consequently, the lossless two-port cannot always be realized with reciprocal elements only. In such a case, howcver, it can be realized using ideal gyrators.

Conditions are also found for the case when an $\mathrm{m}$ variable PRF is realizable both by a $p_{i}$-variable lossless two-port network with a driving-point impedance termination $Z_{01}\left(p_{1}, \cdots, p_{i-1}, p_{i+1}, \cdots, p_{m}\right)$ as well as by a $p_{j}$-variable lossless two-port network with a driving-point impedance termination $Z_{02}\left(p_{1}, \cdots, p_{j-1}, p_{j+1}, \cdots, p_{m}\right)$. Some special cases are also discussed where both the $p_{i^{-}}$and $p_{j^{-}}$ variable two-port networks reduce to either simple series or simple shunt branches. Numerical examples are given 